ANNALES

POLONICI MATHEMATICI

LXXVII.2 (2001)

\title{
On shape and multiplicity of solutions for a singularly perturbed Neumann problem
}

\author{
by J. Chabrowski (Brisbane), Peter J. Watson (Brisbane) \\ and JiAnfu YANG (Wuhan and Campinas)
}

\begin{abstract}
We investigate the effect of the topology of the boundary $\partial \Omega$ and of the graph topology of the coefficient $Q$ on the number of solutions of the nonlinear Neumann problem $\left(1_{d}\right)$.
\end{abstract}

1. Introduction. In this paper we investigate the existence of solutions for the Neumann problem

$$
\left\{\begin{array}{l}
-d^{2} \Delta u+u=Q(y) u^{p}, \quad y \in \Omega, \\
u>0 \text { for } y \in \Omega, \quad \frac{\partial u(y)}{\partial n}=0 \quad \text { for } y \in \partial \Omega,
\end{array}\right.
$$

where $\Omega \subset \mathbb{R}^{N}$ is a bounded domain with $C^{3}$-boundary $\partial \Omega, d>0$ is a parameter, $1<p<(N+2) /(N-2), N \geq 3$, and $n$ is the unit outer normal to $\partial \Omega$. It is assumed that the coefficient $Q$ is positive, Hölder continuous with exponent $\alpha \in(0,1)$ on $\bar{\Omega}$ and $Q \not \equiv$ Const on $\partial \Omega$.

Problem $\left(1_{d}\right)$ stems from the studies of pattern formation in biology. In particular, it can be viewed as the steady state problem for a chemotactic aggregation model proposed by Keller and Segel [6]. Problem $\left(1_{d}\right)$ also appears in the study of activator-inhibitor systems in biological pattern formation theory due to Gierer and Meinhardt [5]. In the case of $Q(x) \equiv 1$ on $\Omega$ problem $\left(1_{d}\right)$ has an extensive literature [6], [8]-[12], [14], [15]. In [11] and [12] Ni and Takagi proved that for every $d>0$ sufficiently small, problem $\left(1_{d}\right)$ has a nonconstant least energy solution $u_{d}$. The solutions $u_{d}$ with $d$ small exhibit concentration phenomena. Namely, each solution $u_{d}$ attains its unique maximum at $P_{d}$ on the boundary and $P_{d} \rightarrow P_{\circ}$, where $P_{\circ}$ is located

2000 Mathematics Subject Classification: 35J20, 35J25, 35J60.

Key words and phrases: Neumann problem, subcritical Sobolev exponent, multiple solutions. 
on the "most curved" part of $\partial \Omega$, that is, the mean curvature attains its maximum at this point. Wei [15] constructed a solution $u_{d}$ on some energy level which has only one local maximum point $x_{d} \in \partial \Omega$ and $x_{d} \rightarrow x_{\circ}$, where $x_{\circ}$ is a critical point of $H(x)$. He also established a partial converse showing that each nondegenerate critical point $x_{\circ}$ of $H(x)$ generates a solution $u_{d}$ having only one maximum point $x_{d}$, with $x_{d} \rightarrow x_{\circ}$. Li [8] showed that the nondegeneracy assumption can be replaced by $C^{1}$-stability.

The effect of the topology of $\partial \Omega$ on the existence of multiple solutions was studied by Wang [14]. He proved that for small $d>0$ problem $\left(1_{d}\right)$, with $Q(y) \equiv 1$, has at least $\operatorname{cat}(\partial \Omega)$ distinct single-peak solutions, where $\operatorname{cat}(\partial \Omega)$ is the Lusternik-Schnirelman category of $\partial \Omega$.

The main purpose of this work is to investigate the effect of the graph topology of the coefficient $Q$ and the topology of the boundary $\partial \Omega$ on the existence of multiple solutions. It is assumed that the coefficient $Q$ attains its maximum on the boundary $\partial \Omega$. We show the existence of the least energy solutions $u_{d}, d>0$. These solutions achieve their maxima at $x_{d} \in \partial \Omega$ for $d$ sufficiently small and $x_{d} \rightarrow x_{\circ}$, with $Q\left(x_{\circ}\right)=\max _{x \in \bar{\Omega}} Q(x)$. However, if $Q(x)=$ Const on $\partial \Omega$, then the influence of the mean curvature is stronger. In this case solutions concentrate at points on $\partial \Omega$ where $H(x)$ attains its maximum. In Section 4 we extend the result of Wang [14] to the problem $\left(1_{d}\right)$ and show that at levels close to the least energy level problem $\left(1_{d}\right)$ has at least $\operatorname{cat}(\partial \Omega)$ solutions.

Section 5 is devoted to the construction of multi-peak solutions. We aim to show that local minima of the restriction of $Q$ to $\partial \Omega$ generate multi-peak solutions. Our approach is a modification of the construction of multi-peak solutions from [4]. In Section 6 we study the effect of the graph topology of the coefficient $Q$ on the existence of multiple solutions. We express the multiplicity of solutions in terms of the Lusternik-Schnirelman category of the set where $Q$ attains its maximum on the boundary $\partial Q$. As in [14] it can be shown that solutions obtained in Section 4 are one-peak solutions, that is, they have at most one local maximum. However, we were unable to locate their concentration points. On the other hand, solutions constructed in Section 6, which are one-peak solutions, have maxima concentrating at points of $\partial \Omega$ where $Q$ attains its maximum.

2. Least energy solutions. Solutions of problem $\left(1_{d}\right)$ will be found as critical points of the variational functional $I_{d}: H^{1}(\Omega) \rightarrow \mathbb{R}$ defined by

$$
I_{d}(u)=\frac{1}{2} \int_{\Omega}\left(d^{2}|\nabla u|^{2}+u^{2}\right) d x-\frac{1}{p+1} \int_{\Omega} Q(x)|u|^{p+1} d x,
$$

where $H^{1}(\Omega)$ is a Sobolev space equipped with the norm 


$$
\|u\|^{2}=\int_{\Omega}\left(|\nabla u(x)|^{2}+u(x)^{2}\right) d x .
$$

It is easy to check that the functional $I_{d}$ has a mountain pass structure:

(i) there exist constants $\varrho>0$ and $\beta>0$ such that $I_{d}(u) \geq \beta$ for $\|u\|=\varrho$;

(ii) there exists $\phi \in H^{1}(\Omega)$, with $\|\phi\|>\varrho$, such that $I_{d}(\phi)<0$;

(iii) $I_{d}$ satisfies the Palais-Smale condition: if $\left\{u_{n}\right\} \subset H^{1}(\Omega)$ is such that $I_{d}\left(u_{n}\right)$ is bounded and $I_{d}^{\prime}\left(u_{n}\right) \rightarrow 0$ in $H^{-1}(\Omega)$ then $\left\{u_{n}\right\}$ is relatively compact in $H^{1}(\Omega)$.

Let $\Gamma=\left\{\gamma \in C\left([0,1], H^{1}(\Omega)\right): \gamma(0)=0, \gamma(1)=\phi\right\}$. By the mountain pass principle [1] for each $d>0$ there exists $u_{d} \in H^{1}(\Omega)$ such that

$$
I_{d}\left(u_{d}\right)=c_{d}=\inf _{\gamma \in \Gamma} \max _{t \in[0,1]} I_{d}(\gamma(t)) .
$$

Using the Hopf maximum principle we can assume that $u_{d}>0$ on $\Omega$ (see $[9$, p. 9]). Repeating the argument from $[9$, pp. 12,13$]$ one can show that $c_{d}=O\left(d^{N}\right)$.

Let $P \in \partial \Omega$. In order to examine the behaviour of $u_{d}$ near the boundary $\partial \Omega$ we introduce the diffeomorphism which straightens the boundary portion near the point $P$ (see [10], [11]). We may assume that $P$ is the origin and the inner normal to $\partial \Omega$ at $P$ is pointing in the direction of the positive $x_{N}$-axis. Then there exists a smooth function $\psi_{P}\left(x^{\prime}\right), x^{\prime}=\left(x_{1}, \ldots, x_{N-1}\right)$, defined for $\left|x^{\prime}\right|$ small such that (a) $\psi_{P}(0)=0, \nabla \psi_{P}(0)=0$, and (b) $\partial \Omega \cap \mathcal{N}=$ $\left\{\left(x^{\prime}, x_{N}\right): x_{N}=\psi_{P}\left(x^{\prime}\right)\right\}$ and $\Omega \cap \mathcal{N}=\left\{\left(x^{\prime}, x_{N}\right): x_{N}>\psi_{P}\left(x^{\prime}\right)\right\}$, where $\mathcal{N}$ is a neighbourhood of $P$.

For $y \in \mathbb{R}^{N}$ near 0 , we define a mapping $x=\Phi_{P}(y)=\left(\Phi_{P, 1}(y), \ldots\right.$, $\left.\Phi_{P, N}(y)\right)$ by

$$
\Phi_{P, j}(y)= \begin{cases}y_{j}-y_{N} \frac{\psi_{P}\left(y^{\prime}\right)}{\partial x_{j}}, & j=1, \ldots, N-1, \\ y_{N}+\psi_{P}\left(y^{\prime}\right), & j=N .\end{cases}
$$

Since $\nabla \psi_{P}(0)=0$, we have $\nabla \Phi_{P}(0)=I$. Therefore $\Phi_{P}$ has an inverse mapping $y=\Phi_{P}^{-1}(x)$ for $|x|<\delta^{\prime}$, with $\delta^{\prime}>0$ small. We set $\Psi_{P}(x)=\Phi_{P}^{-1}(x)$. We notice that

$$
\left|\Phi_{P}(y)\right| \leq C|y|
$$

for some constant $C>0$ and small $|y|$. Following the ideas from [12] and [14] we define a comparison function for $I_{d}$. Towards this end let $w_{M}$ be the ground state solution [7] of

$$
\begin{cases}-\Delta u+u=M u^{p} & \text { in } \mathbb{R}^{N} \\ u>0 & \text { on } \mathbb{R}^{N}\end{cases}
$$


By rescaling we have $w_{M}=M^{-1 /(p-1)} w_{1}$, where $w_{1}>0$ is spherically symmetric: $w_{1}(x)=w_{1}(|x|), d w_{1} / d r<0$ for $|x|>0$. It is known that $w_{1}$ and its first order derivatives exponentially decay at infinity, that is,

$$
w_{1}(x),\left|\nabla w_{1}(x)\right| \leq C e^{-\mu|x|} \quad \text { on } \mathbb{R}^{N},
$$

for some constants $C>0$ and $\mu>0$. For $\varrho>0$ we set

$$
\zeta_{\varrho}(t)= \begin{cases}1 & \text { for } 0 \leq t \leq \varrho, \\ 2-\varrho^{-1} t & \text { for } \varrho<t \leq 2 \varrho, \\ 0 & \text { for } t \geq 2 \varrho\end{cases}
$$

and

$$
w_{*}^{M}(z)=\zeta_{k / d}(|z|) w_{M}(z),
$$

where $k>0$ is chosen so that the domain of definition of $\Phi_{P}$ contains the ball $B_{3 k}=B(0,3 k)$. Further, let $D_{j}=\Phi_{P}\left(B_{j k}^{+}\right)$for $j=1,2$, with $B_{r}^{+}=B_{r} \cap \mathbb{R}_{+}^{N}$. We observe that $D_{1} \subset D_{2} \subset \Omega$. We define a comparison function by

$$
\phi_{d}^{M}(x)= \begin{cases}w_{*}^{M}\left(\Psi_{P}(x) / d\right) & \text { in } D_{2}, \\ 0 & \text { elsewhere. }\end{cases}
$$

In what follows we shall use for a fixed $P \in \bar{\Omega}$ the following functional:

$$
I_{Q(P)}(u)=\frac{1}{2} \int_{\Omega}\left(|\nabla u|^{2}+u^{2}\right) d x-\frac{Q(P)}{p+1} \int_{\Omega}|u|^{p+1} d x .
$$

3. Behaviour of the least energy solutions for small $d>0$. We begin by estimating $I_{d}$ at $\phi_{d}$.

Lemma 1. Suppose $P \in \partial \Omega$. Let $\phi_{d}=\phi_{d}^{Q(P)}$ be defined by (5). Then

$$
M\left[\phi_{d}\right]=\sup _{t \geq 0} I_{d}\left(t \phi_{d}\right)=\frac{d^{N}}{2} I_{Q(P)}\left(w_{Q(P)}\right) A_{d},
$$

where $A_{d}>0$ and $A_{d} \rightarrow 1$ as $d \rightarrow 0$.

Proof. We commence by observing that there exists $t_{\circ}(d)>0$ (unique) such that

$$
M\left[\phi_{d}\right]=I_{d}\left(t_{\circ}(d) \phi_{d}\right) .
$$

To simplify we assume that $P=$ the origin of the coordinates. Since

$$
\left.\frac{d}{d t} I_{d}\left(t \phi_{d}\right)\right|_{t=t_{\circ}(d)}=0
$$

we have

$$
t_{\circ}(d)=\left(\frac{\int_{\Omega}\left(d^{2}\left|\nabla \phi_{d}\right|^{2}+\phi^{2}\right) d x}{\int_{\Omega} Q(x) \phi_{d}^{p+1} d x}\right)^{1 /(p-1)} .
$$


Hence

(6)

$$
\begin{aligned}
& I_{d}\left(t_{\circ}(d) \phi_{d}\right) \\
& \quad=\frac{p-1}{2(p+1)}\left(\frac{\int_{\Omega}\left(d^{2}\left|\nabla \phi_{d}\right|^{2}+\phi_{d}^{2}\right) d x}{\int_{\Omega} Q(x) \phi_{d}^{p+1} d x}\right)^{2 /(p-1)} \int_{\Omega}\left(d^{2}\left|\nabla \phi_{d}\right|^{2}+\phi_{d}^{2}\right) d x .
\end{aligned}
$$

It follows from (3.14) and (3.15) in [12] that

$$
\begin{aligned}
d^{2} \int_{\Omega}\left|\nabla \phi_{d}\right|^{2} d x & =d^{N}\left(\int_{\mathbb{R}_{+}^{N}}\left(w_{Q(0)}^{\prime}\right)^{2} d x+O(d)\right) \\
\int_{\Omega} \phi_{d}^{p+1} d x & =d^{N}\left(\int_{\mathbb{R}_{+}^{N}} w_{Q(0)}^{p+1} d x+O(d)\right) \\
\int_{\Omega} \phi_{d}^{2} d x & =d^{N}\left(\int_{\mathbb{R}_{+}^{N}} w_{Q(0)}^{2} d x+O(d)\right)
\end{aligned}
$$

Using (8) we now write

$$
\begin{aligned}
\int_{\Omega} Q(x) \phi_{d}^{p+1} d x & =Q(0) \int_{\Omega} \phi_{d}^{p+1} d x+\int_{\Omega}(Q(x)-Q(0)) \phi_{d}^{p+1} d x \\
& =Q(0) d^{N}\left(\int_{\mathbb{R}_{+}^{N}} w_{Q(0)}^{p+1} d x+O(d)\right)+\int_{\Omega}(Q(x)-Q(0)) \phi_{d}^{p+1} d x .
\end{aligned}
$$

The second integral on the right side can be estimated using (A.3) of Lemma A.1 in [12]:

$$
\begin{aligned}
\int_{\Omega} \mid Q(x)- & Q(0) \mid \phi_{d}^{p+1} d x \\
& \leq L \int_{D_{2}}|x|^{\alpha} w_{*}^{Q(0)}\left(\frac{\Psi(x)}{d}\right)^{p+1} d x \\
& =L \int_{B_{2 k}^{+}}|\Phi(y)|^{\alpha} w_{*}^{Q(0)}\left(\frac{y}{d}\right)^{p+1} \operatorname{det}|D \Phi(y)| d y \\
& \leq L C d^{N+\alpha} \int_{B_{2 R}}|y|^{\alpha} w_{*}^{Q(0)}(y)^{p+1}\left(1-\bar{\alpha} d y_{N}+O\left(d^{2}|y|^{2}\right)\right) d y
\end{aligned}
$$

where $\bar{\alpha}=\Delta \Psi(0)$ and $C$ is a constant from (2) and $L$ is a Hölder constant for $Q$. Using the fact that $w_{1}(z) \leq C e^{-\mu|z|}$ on $\mathbb{R}^{N}$, we derive from the last two estimates that

$$
\int_{\Omega} Q(x) \phi_{d}^{p+1} d x=d^{N}\left(Q(0) \int_{\mathbb{R}_{+}^{N}} w_{Q(0)}^{p+1} d x+O\left(d^{\alpha}\right)\right) .
$$


The result follows from (6), (7), (9) and (10) with

$$
A_{d}=\left(\frac{\int_{\mathbb{R}_{+}^{N}}\left(\left|\nabla w_{Q(0)}\right|^{2}+w_{Q(0)}^{2}\right) d x+O(d)}{\int_{\mathbb{R}_{+}^{N}} Q(0) w_{Q(0)}^{p+1} d x+O\left(d^{\alpha}\right)}\right)^{2 /(p-1)} .
$$

Lemma 2. If $u_{d}$ attains its local maximum at $x_{d} \in \bar{\Omega}$, then

$$
u_{d}\left(x_{d}\right) \geq M^{-1 /(p-1)},
$$

where $M=\max _{x \in \bar{\Omega}} Q(x)$. Also, there exists a constant $\eta>0$ independent of $x_{d}$ and $d$ such that $u_{d}(x) \geq \eta$ for $x \in B\left(x_{d}, d\right) \cap \Omega$ if $d$ is sufficiently small.

Proof. Suppose that $u_{d}\left(x_{d}\right)<M^{-1 /(p-1)}$. If $x_{d} \in \Omega$, then

$$
d^{2} \Delta u_{d}=u_{d}-Q(x) u_{d}^{p}=u_{d}\left(1-Q(x) u_{d}^{p-1}\right) \geq u_{d}\left(1-M u_{d}^{p-1}\right)>0
$$

in a small ball with centre at $x_{d}$. However, this contradicts the inequality $d^{2} \Delta u_{d}\left(x_{d}\right) \leq 0$. Hence, $x_{d} \in \partial \Omega$ and $u_{d}(x)<u_{d}\left(x_{d}\right)$ for $x \in \Omega$ close to $x_{d}$. According to the Hopf boundary point lemma we have $\partial u_{d}\left(x_{d}\right) / \partial n>0$, which does not match the boundary condition. The second assertion follows from the Harnack inequality (see Lemma 4.3 in [9] and p. 830 in [12]).

We are now in a position to locate the maximum points of $u_{d}$.

Theorem 1. Suppose that $\max _{x \in \partial \Omega} Q(x)=\max _{x \in \bar{\Omega}} Q(x)$. Let $u_{d}\left(P_{d}\right)$ $=\max _{x \in \bar{\Omega}} u_{d}(x)$. Then $P_{d} \in \partial \Omega$ for small $d>0$ and $P_{d} \rightarrow \bar{P}$ with $Q(\bar{P})=$ $\max _{x \in \partial \Omega} Q(x)$.

Proof. We follow some ideas from [12]. The proof will be divided in several steps.

SteP I. There exists a constant $C>0$ such that $\operatorname{dist}\left(P_{d}, \partial \Omega\right) \leq C d$. In the contrary case we can find a decreasing sequence $d_{j} \rightarrow 0$ such that

$$
\varrho_{j}=\frac{\operatorname{dist}\left(P_{j}, \partial \Omega\right)}{d_{j}} \rightarrow \infty
$$

as $j \rightarrow \infty$, where $P_{j}=P_{d_{j}}$. We define a function $v_{j}$ on $B_{\varrho_{j}}$ by

$$
v_{j}(z)=u_{d_{j}}\left(P_{j}+d_{j} z\right) \quad \text { for } z \in B_{\varrho_{j}} .
$$

We may assume that $P_{j} \rightarrow \bar{P}$. Using the Schauder estimates we can show as in [12] that $v_{j} \rightarrow w_{Q(\bar{P})}$ in $C_{\mathrm{loc}}^{2}\left(\mathbb{R}^{N}\right)$, where $w_{Q(\bar{P})}$ is the ground state solution of equation (3) with $M=Q(\bar{P})$. Since $M_{1} \leq Q(x) \leq M_{2}$ on $\bar{\Omega}$ for some constants $0<M_{1}<M_{2}$ and $w_{Q(\bar{P})}(z)=Q(\bar{P})^{-1 /(p-1)} w_{1}(z)$, we see that

$$
w_{Q(\bar{P})}(z),\left|\nabla w_{Q(\bar{P})}(z)\right| \leq C_{\circ} e^{-\mu|z|} \quad \text { on } \mathbb{R}^{N},
$$


for some constants $\mu>0$ and $C_{\circ}>0$ independent of $P$. For the mountain pass level we have the following estimate from below for each $R>0$ :

$$
\begin{aligned}
c_{d_{j}} \geq & \int_{\left|x-P_{j}\right|<d_{j} R} \frac{p-1}{2(p+1)} Q(x) u_{d_{j}}(x)^{p+1} d x \\
= & d_{j}^{N} \int_{|z|<R} \frac{p-1}{2(p+1)} Q\left(P_{j}+d_{j} z\right) v_{j}(z)^{p+1} d z \\
= & d_{j}^{N} \int_{|z|<R} \frac{p-1}{2(p+1)} Q(\bar{P}) w_{Q(\bar{P})}(z)^{p+1} d z \\
& +d_{j}^{N} \int_{|z|<R} \frac{p-1}{2(p+1)} Q\left(P_{j}+d_{j} z\right) v_{j}(z)^{p+1} d z \\
& -d_{j}^{N} \int_{|z|<R} \frac{p-1}{2(p+1)} Q(\bar{P}) w_{Q(\bar{P})}(z)^{p+1} d z \\
= & d_{j}^{N} \int_{|z|<R} \frac{p-1}{2(p+1)} Q(\bar{P}) w_{Q(\bar{P})}(z)^{p+1} d z \\
& +d_{j}^{N} \int_{|z|<R} \frac{p-1}{2(p+1)} Q\left(P_{j}+d_{j} z\right)\left(v_{j}(z)^{p+1}-w_{Q(\bar{P})}(z)^{p+1}\right) d z \\
& +d_{j}^{N} \int_{|z|<R} \frac{p-1}{2(p+1)}\left(Q\left(P_{j}+d_{j} z\right)-Q(\bar{P})\right) w_{Q(\bar{P})}(z)^{p+1} d z .
\end{aligned}
$$

Let us denote the last two integrals on the right side of this inequality by $J_{1}$ and $J_{2}$, respectively. Let $R>0$ be a fixed and large number and set $d_{R}=C_{\circ} e^{-\mu R / 2}$. We choose $j_{\circ}=j_{\circ}(R)$ sufficiently large so that $\left\|v_{j}-w_{Q(\bar{P})}\right\|_{C^{2}(\overline{B(0, R)})} \leq d_{R}$ for $j \geq j_{\circ}$. Thus

$$
\left|J_{1}\right| \leq C_{1} d_{j}^{N} R^{N} d_{R}
$$

for some constant $C_{1}>0$ and all $j \geq j_{\circ}$. Since $Q\left(P_{j}+d_{j} z\right) \rightarrow Q(\bar{P})$ uniformly on $\overline{B(0,2 R)}$, we have

$$
\left|J_{2}\right| \leq C_{2} d_{j}^{N o(1)}
$$

for some constant $C_{2}>0$ and $j \geq j_{\circ}$. Inserting estimates (12) and (13) into (11) we get

$$
c_{d_{j}} \geq d_{j}^{N}\left(\int_{B(0, R)} \frac{p-1}{2(p+1)} Q(\bar{P}) w_{Q(\bar{P})}^{p+1} d x-C_{1} R^{N} d_{R}+o(1)\right) .
$$

Next, we observe that 


$$
\begin{aligned}
\int_{B(0, R)} \frac{p-1}{2(p+1)} & Q(\bar{P}) w_{Q(\bar{P})}(z)^{p+1} d x \\
& =I_{Q(\bar{P})}\left(w_{Q(\bar{P})}\right)-\int_{|z|>R} \frac{p-1}{2(p+1)} Q(\bar{P}) w_{Q(\bar{P})}(z)^{p+1} d z \\
& \geq I_{Q(\bar{P})}\left(w_{Q(\bar{P})}\right)-C_{3} e^{-\mu R}
\end{aligned}
$$

for some constant $C_{3}>0$. Combining the last two estimates we derive the following estimate from below for the level $c_{d_{j}}$ :

$$
c_{d_{j}} \geq d_{j}^{N}\left(I_{Q(\bar{P})}\left(w_{Q(\bar{P})}\right)-C e^{-\mu R}+o(1)\right)
$$

for some constants $\mu>0$ and $C>0$. On the other hand, by Lemma 1 we have

$$
c_{d_{j}} \leq M\left[\phi_{d_{j}}\right] \leq \frac{d_{j}^{N}}{2} I_{Q(P)}\left(w_{Q(P)}\right) A_{d_{j}}
$$

for each $P \in \partial \Omega$. Combining the last two estimates and letting first $j \rightarrow \infty$ and then $R \rightarrow \infty$ we obtain

$$
\frac{p-1}{2(p+1)} \int_{\mathbb{R}^{N}} Q(\bar{P}) w_{Q(\bar{P})}^{p+1} d x \leq \frac{p-1}{4(p+1)} \int_{\mathbb{R}^{N}} Q(P) w_{Q(P)}^{p+1} d x
$$

for each $P \in \partial \Omega$. Since $w_{Q(P)}=Q(P)^{-1 /(p-1)} w_{1}$ for each $P$, we see that this inequality is equivalent to

$$
Q(\bar{P}) \geq 2^{(p-1) / 2} Q(P)
$$

for each $P \in \partial \Omega$. This contradicts the fact that $Q$ achieves its maximum on the boundary.

STEP II. We show that $P_{d} \in \partial \Omega$ for $d>0$ small. Arguing indirectly we may assume that $P_{j}=P_{d_{j}} \in \Omega$ for some decreasing sequence $d_{j} \rightarrow 0$. By Step I, $P_{j} \rightarrow P \in \partial \Omega$ and we assume that $P=0$. Let $y=\Psi(x)$ be a diffeomorphism which straightens the boundary $\partial \Omega$ near $P$. We assume that the closed ball $\bar{B}_{2 k}$ is contained in the domain of definition of $\Phi=\Psi^{-1}$ for some $k>0$ and let $Q_{j}=\Psi\left(P_{j}\right) \in \bar{B}_{k}^{+}$for all $j$. We set $v_{j}(y)=u_{j}(\Phi(y))$ for $y \in \bar{B}_{2 k}^{+}$and we extend $v_{j}$ to $\bar{B}_{2 k}$ by the reflection

$$
\bar{v}_{k}(y)= \begin{cases}v_{k}(y) & \text { if } y \in B_{2 k}^{+}, \\ v_{k}\left(y^{\prime},-y_{N}\right) & \text { if } y \in B_{2 k}^{-}\end{cases}
$$

where $B_{2 k}^{-}=D_{2 k} \cap\left\{y \in \mathbb{R}^{N}: y_{N}<0\right\}$. Finally, we set $w_{j}(z)=\bar{v}_{j}\left(Q_{j}+d_{j} z\right)$ for $z \in \bar{B}_{k / d_{j}}$. Let $Q_{j}=\left(q_{j}^{\prime}, \alpha_{j} d_{j}\right)$, where $q_{j}^{\prime} \in \mathbb{R}^{N-1}$ and $\alpha_{j}>0$. As in [12] using the Schauder estimates one can show that $w_{j} \rightarrow w_{Q(P)}$ in $C_{\text {loc }}^{2}\left(\mathbb{R}^{N}\right)$, where $w_{Q(P)}$ is a ground state solution of $(3)$ with $M=Q(P)$. Here we have used the fact that $Q_{j} \rightarrow 0$. Since the ground state has only one maximum we deduce from Lemma 4.2 in [12], repeating the argument from p. 837 in 
[12], that $w_{j}$ attains only one maximum in a ball $B(0, R)$, where $R>0$ is chosen so that $R>\alpha_{j}$ for all $j$. If $\alpha_{j}>0$, then it follows from the definition of $\bar{v}_{j}$ that $Q_{j}^{*}=\left(q_{j}^{\prime},-\alpha_{j} d_{j}\right)$ is also another local maximum point of $w_{j}$ in $B(0, R)$, which is impossible.

STEP III. We show that $u_{d}$ has at most one local maximum. In the contrary case there exists a decreasing sequence $d_{j}$ such that $u_{d_{j}}$ has two local maxima at $P_{j}$ and $P_{j}^{\prime}$. By the previous part of the proof $P_{j}, P_{j}^{\prime} \in \partial \Omega$. We may assume that $\left|P_{j}-P_{j}^{\prime}\right| / d_{j} \rightarrow \infty$ as $j \rightarrow \infty$, since in the contrary case the scaled function from Step II has two local maxima in the ball $B(0, R)$. Assume $P_{j} \rightarrow \bar{P} \in \partial \Omega$. We now introduce the diffeomorphism $y=\Psi(x)$ which straightens a part of the boundary $\partial \Omega$ around $P_{j}$ and define, as in Step II, $v_{j}, \bar{v}_{j}$ and $w_{j}$. Using the Schauder estimates we show that, up to a subsequence, $w_{j} \rightarrow w_{Q(\bar{P})}$ in $C_{\mathrm{loc}}^{2}\left(\mathbb{R}^{N}\right)$ and $w_{Q(\bar{P})}$ is the ground state solution of (3) with $M=Q(\bar{P})$. Since $u_{j}=u_{d_{j}}$ satisfies (3) we see that

$$
\begin{aligned}
c_{d_{j}} & =\int_{\Omega} \frac{p-1}{2(p+1)} Q(x) u_{j}(x)^{p+1} d x \\
& =\int_{D_{1}} \frac{p-1}{2(p+1)} Q(x) u_{j}(x)^{p+1} d x+\int_{\Omega-D_{1}} \frac{p-1}{2(p+1)} Q(x) u_{j}(x)^{p+1} d x \\
& =I_{1}+I_{2},
\end{aligned}
$$

where $D_{1}=\Phi\left(B_{R d_{j}}\right)$. As in Step I (see also p. 832 in [12]) we check that

$$
I_{1} \geq d_{j}^{N}\left[\int_{B_{k}^{+}} \frac{p-1}{2(p+1)} Q(\bar{P}) w_{Q(\bar{P})}(z)^{p+1} d z-C_{1} R^{N} d_{R}-C_{2} d_{j}\right]
$$

for some constants $C_{1}>0$ and $C_{2}>0$. It follows from Lemma 2 that $u_{j}$ is bounded away from zero on $B_{d_{j}}\left(P_{j}^{\prime}\right) \cap \Omega$, uniformly in $j$. Consequently,

$$
I_{2}=\int_{\Omega-D_{1}} \frac{p-1}{2(p+1)} Q(x) u_{j}(x)^{p+1} d x \geq \eta
$$

for some constant $\eta>0$ independent of $j$. Estimates (14) and (15) give the following estimate of $c_{d_{j}}$ from below:

$$
c_{d_{j}} \geq d_{j}^{N}\left(\frac{1}{2} I_{Q(\bar{P})}\left(w_{Q(\bar{P})}\right)+\eta-C e^{-\mu R}-C_{2} d_{j}\right) .
$$

Here we have used the exponential decay of $w_{Q(\bar{P})}$. On the other hand, by Lemma 1 we have

$$
c_{d_{j}} \leq M\left[\phi_{d_{j}}\right] \leq d_{j}^{N}\left(\frac{1}{2} I_{Q(\bar{P})}\left(w_{Q(\bar{P})}\right)+O\left(d^{\alpha}\right)\right) .
$$

The last estimate contradicts (16) if $d_{j}$ is sufficiently small and $R$ sufficiently large. 
STEP IV. In the final step we show that if $u_{d}\left(P_{d}\right)=\max _{x \in \bar{\Omega}} u_{d}(x)$, then $P_{d} \rightarrow P^{*}$, where $Q\left(P^{*}\right)=\max _{x \in \partial \Omega} Q(x), P^{*} \in \partial \Omega$. Obviously, by the previous steps $P_{d} \in \partial \Omega$. Suppose that $P_{d} \rightarrow P^{*} \in \partial \Omega$. As in the paper [12] (see pp. 837-838 there) we can establish the following estimate of $c_{d}$ from below:

$$
c_{d} \geq d^{N}\left(\frac{1}{2} I_{Q\left(P^{*}\right)}\left(w_{Q\left(P^{*}\right)}\right)-C e^{-\mu R}+o(1)\right),
$$

where $C>0$ and $\mu>0$ are constants independent of $R$ and $o(1) \rightarrow 0$ as $d \rightarrow 0$. Using this and the estimate from Lemma 1 we get

$$
\begin{aligned}
\frac{p-1}{4(p+1)} Q\left(P^{*}\right)^{-1 /(p-1)} & \int_{\mathbb{R}^{N}} w_{1}(z)^{p+1} d z-C e^{-\mu R}+o(1) \\
\leq & \frac{p-1}{4(p+1)} Q(P)^{-1 /(p-1)} \int_{\mathbb{R}^{N}} w_{1}(z)^{p+1} d z+O\left(d^{\alpha}\right)
\end{aligned}
$$

for every $P \in \partial \Omega$. Letting $d \rightarrow 0$ and then $R \rightarrow \infty$ we obtain

$$
Q\left(P^{*}\right)^{-1 /(p-1)} \leq Q(P)^{-1 /(p-1)}
$$

for each $P \in \partial \Omega$ and the claim follows.

Inspection of the proof of Step 1 of Theorem 1 shows that if $Q(x) \leq$ $h Q(y)$ for all $x \in \Omega$ and $y$ in $\partial \Omega$ for some constant $1<h<2^{(p-1) / 2}$, then the points $P_{d}$ where $u_{d}$ achieve their maxima concentrate at a boundary point of $\Omega$. However, it is not clear whether $P_{d} \in \partial \Omega$ for small $d$ under this assumption on $Q$.

If $Q(x)=M$ on $\partial \Omega$ and $Q(x) \leq M$ for all $x \in \Omega$, where $M>0$ is a constant, then according to Theorem $1, u_{d}$ concentrates on the boundary. The question is how to locate a point of $\partial \Omega$ at which the concentration occurs. We show that, under an additional assumption on the behaviour of $Q(x)$ near the boundary, the concentration occurs at a point where the mean curvature of $\partial \Omega$ attains its maximum. We need the following asymptotic formula for $c_{d}$.

Proposition 1. Suppose that $Q(x) \leq M$ on $\bar{\Omega}$ and $Q(x)=M$ on $\partial \Omega$ for some constant $M>0$, and moreover,

$$
|Q(x)-M|=O\left(\operatorname{dist}(x, \partial \Omega)^{k}\right)
$$

for $x$ close to $\partial \Omega$ and some constant $k>1$. Then

$$
c_{d}=d^{N}\left\{I_{M}\left(w_{M}\right)-(N-1) \gamma M^{-1 /(p-2)} H\left(P_{d}\right)+o(d)\right\}
$$

as $d \rightarrow 0$, where $u_{d}\left(P_{d}\right)=\max _{x \in \Omega} u_{d}(x), H(P)$ denotes the mean curvature of $\partial \Omega$ at $P$ and

$$
\gamma=\frac{1}{N+1} \int_{\mathbb{R}_{+}^{N}} w^{\prime}(|z|)^{2} z_{N} d z>0 .
$$

(The quantity $o(d)$ in (18) is independent of $P_{d}$.) 
The proof of Proposition 1 is identical to that of Proposition 2.1 in [11]. In the Appendix we provide a sketch of the proof.

Similarly, we have

$$
M\left[\phi_{d}\right] \leq d^{N}\left\{\frac{1}{2} I_{M}\left(w_{M}\right)-(N-1) \gamma H(P) M^{-2 /(p-1)}+o(d)\right\}
$$

for all $P \in \partial \Omega$.

Theorem 2. Suppose that the assumptions of Proposition 1 hold and let $u_{d}\left(P_{d}\right)=\max _{x \in \Omega} u_{d}(x)$. Then $P_{d} \in \partial \Omega$ for small $d$ and $P_{d} \rightarrow \bar{P}$, with $H(\bar{P})=\max _{P \in \partial \Omega} H(P)$.

Proof. Since $c_{d} \leq M\left[\phi_{d}\right]$, it follows from (18) and (19) that $H\left(P_{d}\right) \geq$ $H(P)+o(d)$ for $d$ sufficiently small and all $P \in \partial \Omega$. Letting $d \rightarrow 0$ we get $H(\bar{P}) \geq H(P)$ for all $P \in \partial \Omega$.

4. Multiple single peak solutions. In this section we calculate a lower bound on the number of single peak solutions to problem $\left(1_{d}\right)$ in terms of the category of $\partial \Omega$. Explicitly, we show there are at least cat $(\partial \Omega)$ distinct nonconstant solutions provided $d$ is sufficiently small.

Assume throughout this section that $Q \in C^{2}(\bar{\Omega})$ and $0<M_{1}=\min _{\Omega} Q(x)$ $<M_{2}=\max _{\bar{\Omega}} Q(x)=\max _{\partial \Omega} Q(x)$.

It is convenient for our purposes to consider a different functional than $I_{d}(u)$ as used in the previous section. We look for minima of the functional

$$
E_{d}(u)=\int_{\Omega}\left(d^{2}|\nabla u|^{2}+u^{2}\right) d x
$$

constrained to the manifold

$$
V_{1}^{Q}(\Omega)=\left\{u \in H^{1}(\Omega): \int_{\Omega} Q(x)|u|^{p+1} d x=1\right\} .
$$

One may check that if $u$ is a critical point of $E_{d}$ on $V_{1}^{Q}(\Omega)$ then $v=$ $\left[E_{d}(u)\right]^{1 /(p-1)} u$ is a solution of $\left(1_{d}\right)$. Furthermore, we have the following relation between $I_{d}$ and $E_{d}$ :

$$
I_{d}(v)=\frac{p-1}{2(p+1)}\left[E_{d}(u)\right]^{(p+1) /(p-1)} .
$$

Therefore, an absolute minimum of $E_{d}$ corresponds to the least energy of $I_{d}$.

Let

$$
c_{d}=\min _{u \in V_{1}^{Q}(\Omega)} E_{d}(u),
$$

which is easily seen to be achieved on $V_{1}^{Q}(\Omega)$. Also, a critical point of $E_{d}$ with absolute minimum critical value corresponds to a critical point of $I_{d}$ and therefore a least energy solution of $\left(1_{d}\right)$. 
There exists a one-to-one correspondence between the solutions of $\left(1_{d}\right)$ of arbitrary sign, and the solutions of the rescaled problem

$$
\begin{cases}-\Delta u+u=Q_{d}(x) u^{p}, & x \in \Omega_{1 / d}, \\ \frac{\partial u(x)}{\partial n}=0, & x \in \partial \Omega_{1 / d},\end{cases}
$$

where $\Omega_{1 / d}=\left\{x \in \mathbb{R}^{N}: d x \in \Omega\right\}$ and $Q_{d}(x)=Q(d x)$. This correspondence is given by

$$
\sigma(u)(x)=d^{N /(p+1)} u(d x) .
$$

The functional associated with problem $\left(1_{1 / d}\right)$ is

$$
\widetilde{E}_{1 / d}(u)=\int_{\Omega_{1 / d}}\left(|\nabla u|^{2}+u^{2}\right) d x
$$

for $u \in V_{1}^{Q}\left(\Omega_{1 / d}\right)=\left\{u \in H^{1}\left(\Omega_{1 / d}\right): \int_{\Omega_{1 / d}} Q_{d}(x)|u|^{p+1} d x=1\right\}$.

By direct computation as shown in [14], Lemma 1.1, we have the following

Lemma 3. For any $u \in V_{1}^{Q}(\Omega)$,

$$
\widetilde{E}_{1 / d}[\sigma(u)]=d^{-N(p-1) /(p+1)} E_{d}(u)
$$

and therefore

$$
\min _{V_{1}^{Q}\left(\Omega_{1 / d}\right)} \widetilde{E}_{1 / d}=d^{-N(p-1) /(p+1)} \min _{V_{1}^{Q}(\Omega)} E_{d}(u) .
$$

The following notation is needed: for $\alpha>0$ and $r \geq 1$ define

$$
\begin{aligned}
V_{\alpha}\left(\Omega_{r}\right) & =\left\{u \in H^{1}\left(\Omega_{r}\right): \int_{\Omega_{r}}|u|^{p+1} d x=\alpha\right\}, \\
V_{\alpha}^{Q}\left(\Omega_{r}\right) & =\left\{u \in H^{1}\left(\Omega_{r}\right): \int_{\Omega_{r}} Q_{1 / r}(x)|u|^{p+1} d x=\alpha\right\}, \\
m(r, \alpha) & =\min _{u \in V_{\alpha}\left(\Omega_{r}\right)} \widetilde{E}_{r}(u), \\
m_{Q}(r, \alpha) & =\min _{u \in V_{\alpha}^{Q}\left(\Omega_{r}\right)} \widetilde{E}_{r}(u), \\
m(+, \alpha) & =\min _{\{}\left\{\int_{\mathbb{R}_{+}^{N}}\left(|\nabla u|^{2}+u^{2}\right) d x: u \in H^{1}\left(\mathbb{R}_{+}^{N}\right), \int_{\mathbb{R}_{+}^{N}}|u|^{p+1} d x=\alpha\right\}, \\
m(\infty, \alpha) & =\min \left\{\int_{\mathbb{R}^{N}}\left(|\nabla u|^{2}+u^{2}\right) d x: u \in H^{1}\left(\mathbb{R}^{N}\right), \int_{\mathbb{R}^{N}}|u|^{p+1} d x=\alpha\right\} .
\end{aligned}
$$

Let $w_{1}$ be the ground state solution of (3) with $M=1$ and let $\widetilde{w}=$ $w_{1} /\left\|w_{1}\right\|_{L^{p+1}}$. The following result is Lemma 1.2 of [14]. 
Lemma 4. For $r \geq 1$ and $\alpha>0$ :

(i) $m(\infty, 1)=\int_{\mathbb{R}^{N}}\left(|\nabla \widetilde{w}|^{2}+|\widetilde{w}|^{2}\right) d x$;

(ii) $m(r, \alpha)=\alpha^{2 /(p+1)} m(r, 1)$ where $r$ may be + or $\infty$ as well;

(iii) $m(\infty, 2)=2 m(+, 1)$.

Now we define a comparison function in order to find an asymptotic estimate of $c_{d}$. Let $\varrho>0$ be fixed throughout this section (different from $\varrho$ in Section 2) such that the neighbourhood

$$
N_{\varrho}(\partial \Omega)=\left\{x \in \mathbb{R}^{N}: \operatorname{dist}(x, \partial \Omega)<\varrho\right\}
$$

is homotopic to $\partial \Omega$. Define

$$
\eta(t)= \begin{cases}1 & \text { for } 0 \leq t \leq 1 \\ 0 & \text { for } t \geq 2\end{cases}
$$

and $\left|\eta^{\prime}(t)\right| \leq 2$. For $P \in \partial \Omega$ let $w_{Q(P)} \equiv w_{P}$ be the ground state solution of (3) with $M=Q(P)$ and set

$$
\psi_{d}(P)(x)=\eta\left(\frac{|x-P|}{\varrho}\right) w_{P}\left(\frac{x-P}{d}\right)
$$

for $x \in \bar{\Omega}$. The comparison function is defined as

$$
\phi_{d}(P)(x)=\frac{\psi_{d}(P)(x)}{Q^{1 /(p+1)}(x)\left\|\psi_{d}(P)\right\|_{L^{p+1}(\Omega)}} .
$$

Clearly, $\phi_{d}(P) \in V_{1}^{Q}(\Omega)$. For $u \in V_{1}^{Q}(\Omega)$ we define a "barycenter" function as

$$
\beta(u)=\int_{\Omega} Q(x)|u|^{p+1} x d x .
$$

It is clear that $\beta(u) \in \overline{\operatorname{conv}}(\Omega)$ for $u \in V_{1}^{Q}(\Omega)$. Furthermore, using the arguments of Proposition 2 below, one may show

$$
\beta\left(\phi_{d}(P)\right)=P+o(1) \quad \text { as } d \rightarrow 0 .
$$

Proposition 2. For $P \in \partial \Omega$,

$$
E_{d}\left(\phi_{d}(P)\right)=d^{N(p-1) /(p+1)}\left(m\left(+, \frac{1}{Q(P)}\right)+o(1)\right)
$$

as $d \rightarrow 0$.

Proof. Upon substitution and expansion we obtain

$$
\begin{aligned}
E_{d}\left(\phi_{d}(P)\right) & =\frac{1}{\left\|\psi_{d}(P)\right\|_{L^{p+1}}^{2}} \int_{\Omega}\left(d^{2}\left|\nabla\left(\frac{\psi_{d}}{Q^{1 /(p+1)}}\right)\right|^{2}+\left|\frac{\psi_{d}}{Q^{1 /(p+1)}}\right|^{2}\right) d x \\
& =\frac{1}{\left\|\psi_{d}\right\|_{L^{p+1}}^{2}}\left(I_{1}+I_{2}+I_{3}\right),
\end{aligned}
$$


where

$$
\begin{aligned}
I_{1}= & \int_{\Omega} \frac{\eta^{2}(|x-P| / \varrho)}{Q^{2 /(p+1)}}\left(\left|\nabla w_{P}\left(\frac{x-P}{d}\right)\right|^{2}+w_{P}^{2}\left(\frac{x-P}{d}\right)\right) d x \\
I_{2}= & d \int_{\Omega}\left[\frac{2}{\varrho Q^{2 /(p+1)}} \eta w_{P} \nabla \eta \cdot \nabla w_{P}\right. \\
& \left.+2 \eta^{2} w_{P} \frac{1}{Q^{1 /(p+1)}} \nabla w_{P} \cdot \nabla\left(\frac{1}{Q^{1 /(p+1)}}\right)\right] d x \\
I_{3}= & d^{2} \int_{\Omega}\left[\frac{1}{\varrho^{2} Q^{2 /(p+1)}}|\nabla \eta|^{2} w_{P}^{2}+\eta^{2} w_{P}^{2}\left|\nabla \frac{1}{Q^{1 /(p+1)}}\right|^{2}\right. \\
& \left.+\frac{2}{\varrho Q^{1 /(p+1)}} \eta w_{P}^{2} \nabla \eta \cdot \nabla\left(\frac{1}{Q^{1 /(p+1)}}\right)\right] d x
\end{aligned}
$$

where we have dropped the arguments of $\eta$ and $w_{P}$ in $I_{2}$ and $I_{3}$ for clarity. We examine each integral in turn.

Using the substitution $z=(x-P) / d$ we obtain

$$
I_{1}=d^{N} \int_{(\Omega-P)_{1 / d}} \frac{\eta^{2}(|z| d / \varrho)}{Q(d z+P)^{2 /(p+1)}}\left(\left|\nabla w_{P}(z)\right|^{2}+w_{P}(z)^{2}\right) d z .
$$

As $w$ and $|\nabla w|$ decay exponentially and $Q$ is bounded, for any $\varepsilon>0$ there exists $R>0$ such that

$$
\int_{(\Omega-P)_{1 / d} \cap(|z| \geq R)} \frac{\eta^{2}}{Q^{2 /(p+1)}}\left(\left|\nabla w_{P}\right|^{2}+w_{P}^{2}\right) d z \leq \frac{\varepsilon}{2} .
$$

For this $R,(\Omega-P)_{1 / d} \cap(|z| \leq R) \rightarrow B_{R}^{+}(0)$ in measure as $d \rightarrow 0$. By the mean value theorem we find that

$$
Q(d z+P)^{2 /(p+1)}=Q(P)^{2 /(p+1)}+O(d) .
$$

Choosing $d<\varrho / R$ sufficiently small we get

$$
\left|\int_{(\Omega-P)_{1 / d} \cap(|z| \leq R)} \frac{\left|\nabla w_{P}\right|^{2}+w_{P}^{2}}{Q(P+d z)^{2 /(p+1)}} d z-\int_{B_{R}^{+}} \frac{\left|\nabla w_{P}\right|^{2}+w_{P}^{2}}{Q(P)^{2 /(p+1)}} d x\right| \leq \frac{\varepsilon}{2}
$$

and therefore

$$
I_{1}=d^{N}\left(\int_{\mathbb{R}_{+}^{N}} \frac{\left|\nabla w_{P}\right|^{2}+w_{P}^{2}}{Q(P)^{2 /(p+1)}} d x+o(1)\right) .
$$

Using the same substitution as above we estimate $I_{2}$ to be 


$$
\begin{aligned}
I_{2}= & d^{N} \int_{(\Omega-P)_{1 / d}} d\left[\frac{2 \eta w_{P}}{\varrho Q(P+d z)^{2 /(p+1)}} \nabla \eta \cdot \nabla w_{p}\right. \\
& \left.+\frac{2 \eta^{2} w_{P}}{Q(P+d z)^{2 /(p+1)}} \nabla w_{P} \cdot \nabla\left(\frac{1}{Q(P+d z)^{1 /(p+1)}}\right)\right] d z \\
\leq & d^{N}\left[\int_{(\Omega-P)_{1 / d} \cap(|z| \leq \varrho / d)} d \frac{2 w_{P}}{Q(P+d z)^{1 /(p+1)}} \nabla w_{P} \cdot \nabla\left(\frac{1}{Q^{1 /(p+1)}}\right) d z\right. \\
& +\int_{(\Omega-P)_{1 / d} \cap(\varrho / d \leq|z| \leq 2 \varrho / d)} d\left[\frac{4 w_{P}}{\varrho Q(P+d z)^{2 /(p+1)}}\left|\nabla w_{P}\right|\right. \\
& \left.\left.+\frac{2 w_{p}}{Q(P+d z)^{1 /(p+1)}} \nabla w_{P} \cdot \nabla\left(\frac{1}{Q^{1 /(p+1)}}\right)\right] d z\right] .
\end{aligned}
$$

Since $w_{P}$ and $\left|\nabla w_{P}\right|$ decay exponentially and the terms involving $Q$ are bounded independently of $d$, we have

$$
I_{2} \leq d^{N}(o(1))
$$

Precisely the same reasoning shows that

$$
I_{3} \leq d^{N}(o(1))
$$

and so

$$
\begin{aligned}
\int_{\Omega}\left(d^{2}\left|\nabla\left(\frac{\psi_{d}}{Q^{1 /(p+1)}}\right)\right|^{2}+\left|\frac{\psi_{d}}{Q^{1 /(p+1)}}\right|^{2}\right) d x \\
=d^{N}\left(\int_{\mathbb{R}_{+}^{N}} \frac{\left|\nabla w_{P}\right|^{2}+w_{P}^{2}}{Q(P)^{2 /(p+1)}} d x+o(1)\right) .
\end{aligned}
$$

Now we estimate $\left\|\psi_{d}\right\|_{L^{p+1}}^{2}$ :

$$
\begin{aligned}
\left\|\psi_{d}\right\|_{L^{p+1}}^{2}= & {\left[\int_{\Omega} \eta^{p+1}\left(\frac{|x-P|}{\varrho}\right) w_{P}^{p+1}\left(\frac{x-P}{d}\right) d x\right]^{2 /(p+1)} } \\
= & d^{2 N /(p+1)}\left[\int_{(\Omega-P)_{1 / d}} \eta^{p+1}\left(\frac{|z| d}{\varrho}\right) w_{P}^{p+1}(z) d z\right]^{2 /(p+1)} \\
= & d^{2 N /(p+1)}\left[\int_{(\Omega-P)_{1 / d} \cap(|z| \leq \varrho / d)} w_{P}^{p+1}(z) d z\right. \\
& \left.+\int_{(\Omega-P)_{1 / d} \cap(\varrho / d \leq|z| \leq 2 \varrho / d)} \eta^{p+1}\left(\frac{|x-P|}{\varrho}\right) w_{P}^{p+1}(z) d z\right]^{2 /(p+1)}
\end{aligned}
$$




$$
=d^{2 N /(p+1)}\left[\int_{\mathbb{R}_{+}^{N}} w_{P}^{p+1}(z) d z+o(1)\right]^{2 /(p+1)}
$$

hence

$$
\left\|\psi_{d}\right\|_{L^{p+1}}^{2}=d^{2 N /(p+1)}\left\{\left[\int_{\mathbb{R}_{+}^{N}} w_{P}^{p+1}(z) d z\right]^{2 /(p+1)}+o(1)\right\} .
$$

From equations (20) and (21) we find

$$
\begin{aligned}
E_{d}\left(\phi_{d}(P)\right) & =\frac{d^{N}\left(\int_{\mathbb{R}_{+}^{N}} \frac{\left|\nabla w_{P}\right|^{2}+w_{P}^{2}}{Q(P)^{2 /(p+1)}} d x+o(1)\right)}{d^{2 N /(p+1)}\left(\left[\int_{\mathbb{R}_{+}^{N}} w_{P}^{p+1}(x) d x\right]^{2 /(p+1)}+o(1)\right)} \\
& =\frac{d^{N(p-1) /(p+1)}}{Q(P)^{2 /(p+1)}}\left(\frac{\int_{\mathbb{R}_{+}^{N}}\left|\nabla w_{P}\right|^{2}+w_{P}^{2}}{\left(\int_{\mathbb{R}_{+}^{N}} w_{P}^{p+1}\right)^{2 /(p+1)}}+o(1)\right) \\
& =\frac{d^{N(p-1) /(p+1)}}{Q(P)^{2 /(p+1)}}(m(+, 1)+o(1)) \\
& =d^{N(p-1) /(p+1)}\left(m\left(+, \frac{1}{Q(P)}\right)+o(1)\right) \quad \text { as } d \rightarrow 0 .
\end{aligned}
$$

Proposition 3. For $c_{d}=\min _{V_{1}^{Q}(\Omega)} E_{d}(u)$, we have

$$
c_{d}=d^{N(p-1) /(p+1)}\left(m\left(+, \frac{1}{M_{2}}\right)+o(1)\right) .
$$

Proof. As $\phi_{d}(P) \in V_{1}^{Q}(\Omega)$ for any $P \in \partial \Omega$, choosing $P \in \partial \Omega$ with $Q(P)=M_{2}$ we find that

$$
c_{d} \leq \frac{d^{N(p-1) /(p+1)}}{M_{2}^{2 /(p+1)}}(m(+, 1)+o(1)) .
$$

Suppose this inequality is strict, so that

$$
\liminf _{d \rightarrow 0} d^{-N(p-1) /(p+1)} c_{d}<\frac{m(+, 1)}{M_{2}^{2 /(p+1)}} .
$$

Then there is a subsequence $d_{n} \rightarrow 0$ and $u_{n} \in V_{1}^{Q}(\Omega)$ such that

$$
c_{d_{n}} \equiv c_{n}=E_{d_{n}}\left(u_{n}\right)
$$

and

$$
\lim _{n \rightarrow \infty} d_{n}^{-N(p-1) /(p+1)} E_{d_{n}}\left(u_{n}\right)=A<m\left(+, \frac{1}{M_{2}}\right) .
$$

By rescaling $v_{n}=\sigma\left(u_{n}\right)=d_{n}^{N /(p+1)} u_{n}\left(d_{n} x\right)$ we have

$$
\lim _{n \rightarrow \infty} d_{n}^{-N(p-1) /(p+1)} E_{d_{n}}\left(u_{n}\right)=\lim _{n \rightarrow \infty} \widetilde{E}_{1 / d_{n}}\left(v_{n}\right)=A<m\left(+, \frac{1}{M_{2}}\right) .
$$


It is obvious that $A=\lim _{n} m_{Q}\left(1 / d_{n}, 1\right)$ as $v_{n}$ solves the rescaled problem $\left(1_{1 / d_{n}}\right)$. Defining

$$
\mu_{n}=\chi_{n}(x) Q_{n}(x)\left|v_{n}\right|^{p+1},
$$

where $\chi_{n}$ is the characteristic function of $\Omega_{1 / d_{n}} \equiv \Omega_{n}$ and $Q_{n}(x)=Q\left(d_{n} x\right)$, we get

$$
\int_{\mathbb{R}^{N}} \mu_{n}=1
$$

as $v_{n} \in V_{1}^{Q}\left(\Omega_{n}\right)$. Therefore we may apply the Concentration-Compactness principle.

Following the arguments of [14, Lemma 2.1] and replacing the function $v_{n}$ there with $Q_{n}^{1 /(p+1)} v_{n}$ we find that Vanishing of the sequence $\mu_{n}$ does not occur.

If Dichotomy occurs, then there exists $\lambda \in(0,1)$ such that for

$$
\Theta_{n}(t)=\sup _{y \in \mathbb{R}^{N}} \int_{B_{t}(y)} \chi_{n} Q_{n}\left|v_{n}\right|^{p+1} d x \quad \text { and } \quad \Theta(t)=\lim _{n \rightarrow \infty} \Theta_{n}(t),
$$

we have $\lim _{t \rightarrow \infty} \Theta(t)=\lambda$. For any $\varepsilon>0$ there exists $R_{0}$ such that $\Theta\left(R_{0}\right) \geq$ $\lambda-\varepsilon / 6$ and there exist $\left\{y_{n}\right\} \subset \mathbb{R}^{N}$ and $n_{0} \geq 0$ such that for $n \geq n_{0}$,

$$
\Theta_{n}\left(R_{0}\right)-\varepsilon / 6 \leq \int_{B_{R_{0}}\left(y_{n}\right)} \chi_{n} Q_{n}\left|v_{n}\right|^{p+1} d x
$$

and

$$
\left|\Theta\left(R_{0}\right)-\Theta_{n}\left(R_{0}\right)\right| \leq \varepsilon / 6
$$

Therefore

$$
\lambda-\frac{\varepsilon}{6} \leq \Theta\left(R_{0}\right) \leq \Theta_{n}\left(R_{0}\right)+\frac{\varepsilon}{6} \leq \int_{B_{R_{0}}\left(y_{n}\right)} \chi_{n} Q_{n}\left|v_{n}\right|^{p+1} d x+\frac{\varepsilon}{3}
$$

and so

$$
\int_{B_{R_{0}}\left(y_{n}\right)} \chi_{n} Q_{n}\left|v_{n}\right|^{p+1} d x \geq \lambda-\frac{\varepsilon}{2}
$$

for all $n \geq n_{0}$. We may also choose $R_{n} \rightarrow \infty$ such that

$$
\Theta_{n}\left(2 R_{n}\right) \leq \lambda+\varepsilon / 2 .
$$

With $\eta$ as defined above, let $\xi=1-\eta$ and

$$
v_{n}^{1}(x)=\chi_{n}(x) \eta\left(\frac{\left|x-y_{n}\right|}{R_{0}}\right) v_{n}(x), \quad v_{n}^{2}(x)=\chi_{n}(x) \xi\left(\frac{\left|x-y_{n}\right|}{R_{n}}\right) v_{n}(x) .
$$

It is standard to show that

$$
\left|1-\int_{\mathbb{R}^{N}} Q_{n}\left(\left|v_{n}^{1}\right|^{p+1}+\left|v_{n}^{2}\right|^{p+1}\right) d x\right| \leq \varepsilon
$$


for all $n \geq n_{0}$. Thus for all $n \geq n_{0}$,

$$
\left.\left|\int_{\mathbb{R}^{N}} Q_{n}\right| v_{n}^{1}\right|^{p+1} d x-\lambda \mid \leq \frac{\varepsilon}{2} \quad \text { and }\left.\left|\int_{\mathbb{R}^{N}} Q_{n}\right| v_{n}^{1}\right|^{p+1} d x-(1-\lambda) \mid \leq \frac{\varepsilon}{2} .
$$

Hence

$$
\begin{array}{ll}
\int_{\mathbb{R}^{N}} Q_{n}\left|v_{n}^{1}\right|^{p+1} d x=\lambda+\varepsilon_{n}^{1} & \text { with }\left|\varepsilon_{n}^{1}\right| \leq \varepsilon, \\
\int_{\mathbb{R}^{N}} Q_{n}\left|v_{n}^{2}\right|^{p+1} d x=1-\lambda-\varepsilon_{n}^{2} & \text { with }\left|\varepsilon_{n}^{2}\right| \leq \varepsilon .
\end{array}
$$

By choosing $R_{0}$ sufficiently large and keeping $\varepsilon$ fixed we get

$$
\int_{\Omega_{n}}\left(\left|\nabla v_{n}\right|^{2}+\left|v_{n}\right|^{2}\right) d x-\int_{\Omega_{n}}\left(\left|\nabla v_{n}^{1}\right|^{2}+\left|v_{n}^{1}\right|^{2}\right) d x-\int_{\Omega_{n}}\left(\left|\nabla v_{n}^{2}\right|^{2}+\left|v_{n}^{2}\right|^{2}\right) d x \geq-2 \varepsilon .
$$

Thus

$$
\begin{aligned}
A= & \lim _{n \rightarrow \infty} \int_{\Omega_{n}}\left(\left|\nabla v_{n}\right|^{2}+\left|v_{n}\right|^{2}\right) d x \\
\geq & \lim _{n \rightarrow \infty}\left(\int_{\Omega_{n}}\left(\left|\nabla v_{n}^{1}\right|^{2}+\left|v_{n}^{1}\right|^{2}\right) d x+\int_{\Omega_{n}}\left(\left|\nabla v_{n}^{2}\right|^{2}+\left|v_{n}^{2}\right|^{2}\right) d x\right)-2 \varepsilon \\
\geq & \lim _{n \rightarrow \infty}\left(m_{Q}\left(1 / d_{n}, \lambda+\varepsilon_{n}^{1}\right)+m_{Q}\left(1 / d_{n}, 1-\lambda+\varepsilon_{n}^{2}\right)\right)-2 \varepsilon \\
\geq & \lim _{n \rightarrow \infty}\left((\lambda-\varepsilon)^{2 /(p+1)} m_{Q}\left(1 / d_{n}, 1\right)\right. \\
& \left.+(1-\lambda-\varepsilon)^{2 /(p+1)} m_{Q}\left(1 / d_{n}, 1\right)\right)-2 \varepsilon \\
= & (\lambda-\varepsilon)^{2 /(p+1)} A+(1-\lambda-\varepsilon)^{2 /(p+1)} A-2 \varepsilon .
\end{aligned}
$$

Now $A>0$ from the following reasoning: as the embedding $H^{1}\left(\Omega_{n}\right) \rightarrow$ $L^{p+1}\left(\Omega_{n}\right)$ is compact, there is a constant depending only on the cone condition of $\Omega_{n}$ such that $\left\|v_{n}\right\|_{L^{p+1}}^{p+1} \leq \widetilde{c}_{n} \widetilde{E}_{1 / d_{n}}\left(v_{n}\right)$. As the cone condition is independent of $n$, we have $\widetilde{c}_{n} \equiv \widetilde{c}$. As $v_{n} \in V_{1}^{Q}\left(\Omega_{n}\right)$ it is trivial to see that $\left\|v_{n}\right\|_{L^{p+1}}^{p+1} \geq 1 / M_{2}>0$. Thus $\widetilde{E}_{1 / d_{n}}\left(v_{n}\right) \geq \widetilde{c} / M_{2}$ and $A=\lim _{n} \widetilde{E}_{1 / d_{n}}\left(v_{n}\right)>0$.

So dividing the final equation in (22) by $A$ gives

$$
1 \geq(\lambda-\varepsilon)^{2 /(p+1)}+(1-\lambda-\varepsilon)^{2 /(p+1)}-2 \varepsilon
$$

and letting $\varepsilon \rightarrow 0$ we get the contradiction

$$
1 \geq \lambda^{2 /(p+1)}+(1-\lambda)^{2 /(p+1)}>1 .
$$

Therefore Dichotomy does not occur.

With Vanishing and Dichotomy ruled out the sequence $\mu_{n}$ must be tight, so there exists a sequence $\left\{y_{n}\right\} \subset \mathbb{R}^{N}$ such that for any $\varepsilon>0$ there exists $R>0$ such that

$$
\int_{B_{R}\left(y_{n}\right)} \chi_{n} Q_{n}\left|v_{n}\right|^{p+1} d x \geq 1-\varepsilon
$$


Assertion 1. There exists $C>0$ such that $\operatorname{dist}\left(y_{n}, \partial \Omega_{n}\right) \leq C$.

If not, then $\operatorname{dist}\left(y_{n}, \partial \Omega_{n}\right) \rightarrow \infty$. From (23), $y_{n} \in \Omega_{n}$. For $\varepsilon>0$ fixed, let $R>0$ be such that (23) holds. Choose $n$ large such that $B_{2 R}\left(y_{n}\right) \subset \Omega_{n}$ and define

$$
w_{n}(x)=\eta\left(\frac{\left|x-y_{n}\right|}{R}\right) v_{n}(x)
$$

which clearly belongs to $H^{1}\left(\Omega_{n}\right)$. Choosing $R$ larger if necessary, we have

$$
\int_{\Omega_{n}}\left(\left|\nabla v_{n}\right|^{2}+\left|v_{n}\right|^{2}\right) d x-\int_{\Omega_{n}}\left(\left|\nabla w_{n}\right|^{2}+\left|w_{n}\right|^{2}\right) d x \geq-2 \varepsilon .
$$

Let

$$
\lambda_{n} \equiv \int_{\mathbb{R}^{N}} \chi_{n} Q_{n}\left|w_{n}\right|^{p+1} d x \geq 1-\varepsilon
$$

and define

$$
\bar{Q}_{n}=\frac{\lambda_{n}}{\int_{\mathbb{R}^{N}}\left|w_{n}\right|^{p+1} d x} .
$$

Then $\bar{Q}_{n} \in\left[M_{1}, M_{2}\right]$ and so

$$
\begin{aligned}
\int_{\Omega_{n}}\left(\left|\nabla v_{n}\right|^{2}+\left|v_{n}\right|^{2}\right) d x & \geq \int_{\mathbb{R}^{N}}\left(\left|\nabla w_{n}\right|^{2}+\left|w_{n}\right|^{2}\right) d x-2 \varepsilon \\
& \geq m\left(\infty, \frac{\lambda_{n}}{\bar{Q}_{n}}\right)-2 \varepsilon=\frac{\lambda_{n}^{2 /(p+1)}}{\bar{Q}_{n}^{2 /(p+1)}} m(\infty, 1)-2 \varepsilon \\
& \geq \frac{(1-\varepsilon)^{2 /(p+1)}}{\bar{Q}_{n}^{2 /(p+1)}} 2^{(p-1) /(p+1)} m(+, 1)-2 \varepsilon \\
& \geq \frac{(1-\varepsilon)^{2 /(p+1)}}{M_{2}^{2 /(p+1)}} 2^{(p-1) /(p+1)} m(+, 1)-2 \varepsilon \\
& =(1-\varepsilon)^{2 /(p+1)} 2^{(p-1) /(p+1)} m\left(+, \frac{1}{M_{2}}\right)-2 \varepsilon .
\end{aligned}
$$

Letting $n \rightarrow \infty$ and $\varepsilon \rightarrow 0$ gives

$$
m\left(+, \frac{1}{M_{2}}\right)>A \geq 2^{(p-1) /(p+1)} m\left(+, \frac{1}{M_{2}}\right),
$$

a contradiction. Therefore there exists $C>0$ such that

$$
\operatorname{dist}\left(y_{n}, \partial \Omega_{n}\right) \leq C .
$$

Choose $\bar{t}_{n} \in \partial \Omega_{n}$ with $\operatorname{dist}\left(y_{n}, \bar{t}_{n}\right) \leq C$ and let $t_{n}=d_{n} \bar{t}_{n} \in \partial \Omega$. Let $T_{n}$ be a unitary matrix such that $\widetilde{\Omega}_{n}=T_{n}\left(\Omega_{n}-\bar{t}_{n}\right)$ has $y^{N}$ as an inner normal direction to $\partial \widetilde{\Omega}_{n}$ at 0 .

We need the following result of [14] (Assertion 3): 
Assertion 2. For any fixed $R_{1}>0$, as $n \rightarrow \infty$,

$$
T_{n}\left(\Omega_{n}-\bar{t}_{n}\right) \cap B_{R_{1}}(0)
$$

converges to $B_{R_{1}}^{+}(0)$ in the following sense: There exists $K_{1}>0$ and for each $\delta>0$ there exists $n_{\delta}$ such that

$$
\left\{x \in B_{R_{1}}^{+}(0): x^{N} \geq \delta\right\} \subset T_{n}\left(\Omega_{n}-\bar{t}_{n}\right) \quad \text { for all } n \geq n_{\delta},
$$

and

$$
L^{N}\left\{x \in T_{n}\left(\Omega_{n}-\bar{t}_{n}\right) \cap B_{R_{1}}(0): x^{N} \leq \delta\right\} \leq K_{1} \delta
$$

where $L^{N}\{\ldots\}$ is $N$-dimensional Lebesgue measure.

The next result is Assertion 4 of [14]:

ASSERTION 3. $\left\|v_{n}\right\|_{L^{\infty}\left(\Omega_{n}\right)}$ is uniformly bounded independently of $n$.

Now we complete the proof of Proposition 3. As $\mu_{n}$ is tight, for each $\varepsilon>0$ there exists $R>0$ such that (23) holds. Since $\operatorname{dist}\left(y_{n}, \partial \Omega_{n}\right) \leq C$, setting $R_{1}=R+C$ gives

$$
\int_{B_{R_{1}}\left(\bar{t}_{n}\right)} \chi_{n} Q_{n}\left|v_{n}\right|^{p+1} d x \geq 1-\varepsilon .
$$

By Assertions 2 and 3, for the given $\varepsilon$ choose $\delta_{1}>0$ such that

$$
K_{1} \delta_{1} M_{2}\left\|v_{n}\right\|_{L^{\infty}\left(\Omega_{n}\right)}^{p+1}<\varepsilon .
$$

Then there exists $n_{\delta_{1}}$ such that

$$
\begin{aligned}
\int_{B_{R_{1}}\left(\bar{t}_{n}\right)} & \chi_{n} Q_{n}\left|v_{n}\right|^{p+1} d x \\
= & \int_{\left\{x \in B_{R_{1}}^{+}(0): x^{N} \geq \delta_{1}\right\}} Q_{n}\left(T_{n}^{-1} x+\bar{t}_{n}\right)\left|v_{n}\left(T_{n}^{-1} x+\bar{t}_{n}\right)\right|^{p+1} d x \\
& +\int_{\left\{x \in B_{R_{1}}(0): x^{N}<\delta_{1}\right\} \cap T_{n}\left(\Omega_{n}+\bar{t}_{n}\right)} Q_{n}\left(T_{n}^{-1} x+\bar{t}_{n}\right)\left|v_{n}\left(T_{n}^{-1} x+\bar{t}_{n}\right)\right|^{p+1} d x \\
& \geq 1-\varepsilon \quad \text { for all } n \geq n_{\delta_{1}} .
\end{aligned}
$$

As $K_{1} \delta_{1} M_{2}\left\|v_{n}\right\|_{L^{\infty}\left(\Omega_{n}\right)}^{p+1}<\varepsilon$ the second integral is less than $\varepsilon$ by the second part of Assertion 2. Thus

$$
\int_{\left\{x \in B_{R_{1}}^{+}(0): x^{N} \geq \delta_{1}\right\}} Q_{n}\left(T_{n}^{-1} x+\bar{t}_{n}\right)\left|v_{n}\left(T_{n}^{-1} x+\bar{t}_{n}\right)\right|^{p+1} d x \geq 1-2 \varepsilon
$$

for all $n \geq n_{\delta_{1}}$. Define $x_{\delta_{1}}=\left(0, \ldots, 0, \delta_{1}\right)$ and

$$
\widetilde{v}_{n}(x)=\eta\left(|x| / R_{1}\right) v_{n}\left(T_{n}^{-1}\left(x+x_{\delta_{1}}\right)+\bar{t}_{n}\right)
$$


for $x \in \mathbb{R}_{+}^{N}$, which is well defined by the first part of Assertion 2 for $n$ sufficiently large. Choose $R_{1}$ so large to ensure that

$$
\int_{\Omega_{n}}\left(\left|\nabla v_{n}\right|^{2}+v_{n}^{2}\right) d x-\int_{\mathbb{R}_{+}^{N}}\left(\left|\nabla \widetilde{v}_{n}\right|^{2}+\widetilde{v}_{n}^{2}\right) d x \geq-2 \varepsilon
$$

and let

$$
\lambda_{n}=\int_{\left\{x \in B_{R_{1}}^{+}(0): x^{N} \geq \delta_{1}\right\}} Q_{n}\left(T_{n}^{-1} x+\bar{t}_{n}\right)\left|v_{n}\left(T_{n}^{-1} x+\bar{t}_{n}\right)\right|^{p+1} d x \geq 1-2 \varepsilon .
$$

Defining

$$
\bar{Q}_{n}=\frac{\lambda_{n}}{\int_{\mathbb{R}_{+}^{N}}\left|\widetilde{v}_{n}\right|^{p+1} d x}
$$

we get

$$
\begin{aligned}
A & =\lim _{n \rightarrow \infty} \int_{\Omega_{n}}\left(\left|\nabla v_{n}\right|^{2}+v_{n}^{2}\right) d x \geq \lim _{n} \int_{\mathbb{R}^{N}}\left(\left|\nabla \widetilde{v}_{n}\right|^{2}+\widetilde{v}_{n}^{2}\right) d x-2 \varepsilon \\
& \geq \lim _{n} m\left(+, \frac{\lambda_{n}}{\bar{Q}_{n}}\right)-2 \varepsilon=\lim _{n} \frac{\lambda_{n}^{2 /(p+1)}}{\bar{Q}_{n}^{2 /(p+1)}} m(+, 1)-2 \varepsilon \\
& \geq(1-2 \varepsilon)^{2 /(p+1)} \frac{1}{M_{2}^{2 /(p+1)}} m(+, 1)-2 \varepsilon .
\end{aligned}
$$

Letting $\varepsilon \rightarrow 0$ gives the contradiction

$$
m\left(+, \frac{1}{M_{2}}\right)>A \geq m\left(+, \frac{1}{M_{2}}\right)
$$

which completes the proof.

An obvious modification of the arguments used in the above proof reveals the following.

Lemma 5. Let $d_{n} \rightarrow 0$ as $n \rightarrow \infty$ and assume a sequence $v_{n} \in V_{1}^{Q}\left(\Omega_{1 / d_{n}}\right)$ satisfies

$$
\int_{\Omega_{1 / d_{n}}}\left(\left|\nabla v_{n}\right|^{2}+v_{n}^{2}\right) d x \rightarrow m\left(+, \frac{1}{M_{2}}\right)
$$

as $n \rightarrow \infty$. Then there exists a subsequence $v_{n}$, points $y_{n} \in \mathbb{R}^{N}$ and a constant $C$ independent of $n$ such that for $n$ sufficiently large,

$$
\forall \varepsilon \exists R>0 \quad \lim _{n \rightarrow \infty} \int_{B_{R}\left(y_{n}\right) \cap \Omega_{1 / d_{n}}} Q_{n}\left|v_{n}\right|^{p+1} d x \geq 1-\varepsilon
$$

and

$$
\operatorname{dist}\left(y_{n}, \partial \Omega_{n}\right) \leq C .
$$

Now we are in a position to prove 
Proposition 4. For $\varrho>0$ such that $N_{\varrho}(\partial \Omega)$ is homotopic to $\partial \Omega$, there exist $\varepsilon_{1}>0$ and $d_{1}>0$ such that for any $d \in\left(0, d_{1}\right]$,

$$
\beta(u) \in N_{\varrho}(\partial \Omega)
$$

for all $u \in E_{d}^{c_{d}+\varepsilon_{1} d^{N(p-1) /(p+1)}}$.

Proof. Suppose not. Then for each $\varepsilon>0$ and $d>0$ there exists $u \in$ $E_{d}^{c_{d}+\varepsilon d^{N(p-1) /(p+1)}}$ with $\beta(u) \notin N_{\varrho}(\partial \Omega)$. We may choose subsequences $d_{n} \rightarrow$ $0, \varepsilon_{n} \rightarrow 0$ and functions

$$
u_{n} \in E_{d_{n}}^{c_{d_{n}}+\varepsilon_{n} d_{n}^{N(p-1) /(p+1)}}
$$

with $\beta\left(u_{n}\right) \notin N_{\varrho}(\partial \Omega)$. Using Proposition 3 we have

$$
\lim _{n \rightarrow \infty} d_{n}^{-N(p-1) / p+1} E_{d_{n}}\left(u_{n}\right)=m\left(+, \frac{1}{M_{2}}\right) .
$$

Rescaling, we find $v_{n}(x)=d_{n}^{N /(p+1)} u_{n}\left(d_{n} x\right) \in V_{1}^{Q}\left(\Omega_{n}\right)$ and

$$
\lim _{n \rightarrow \infty} \int_{\Omega_{n}}\left(\left|\nabla v_{n}\right|^{2}+v_{n}^{2}\right) d x=m\left(+, \frac{1}{M_{2}}\right) .
$$

By Lemma 5 , there is a subsequence $v_{n}$, points $y_{n} \in \mathbb{R}^{N}$ and $C>0$ such that for any $\varepsilon>0$ there exists $R>0$ such that

$$
\lim _{n \rightarrow \infty} \int_{B_{R}\left(y_{n}\right) \cap \Omega_{n}} Q_{n}\left|v_{n}\right|^{p+1} d x \geq 1-\varepsilon
$$

and

$$
\operatorname{dist}\left(y_{n}, \partial \Omega_{n}\right) \leq C .
$$

We may choose $t_{n} \in \partial \Omega$ such that $\operatorname{dist}\left(y_{n}, t_{n} / d_{n}\right) \leq C$ and $t_{n} \rightarrow t \in \partial \Omega$. Without loss of generality we may assume that $\beta\left(u_{n}\right) \rightarrow 0 \in \mathbb{R}^{N}$.

By direct calculation,

$$
\int_{\Omega_{n}} Q_{n}\left|v_{n}\right|^{p+1} x d x=\frac{\beta\left(u_{n}\right)^{1}}{d_{n}} .
$$

As $\beta\left(u_{n}\right) \notin N_{\varrho}(\partial \Omega)$, we have $t \neq 0$, so without loss of generality, $t=$ $\left(t^{1}, \ldots, t^{N}\right)$ with $t^{1}>0$.

For $\varepsilon>0$ arbitrary, let $R_{1}>0$ such that

$$
\lim _{n \rightarrow \infty} \int_{B_{R_{1}}\left(t_{n} / d_{n}\right) \cap \Omega_{n}} Q_{n}\left|v_{n}\right|^{p+1} d x \geq 1-\varepsilon .
$$

Let $s=\min \left\{y^{1}:\left(y^{1}, \ldots, y^{N}\right) \in \partial \Omega\right\}$. For $n$ large we then have 


$$
\begin{aligned}
\frac{\beta\left(u_{n}\right)^{1}}{d_{n}}= & \int_{\Omega_{n}} Q_{n}\left|v_{n}\right|^{p+1} x^{1} d x \\
= & \int_{\Omega_{n} \cap B_{R_{1}}\left(t_{n} / d_{n}\right)} Q_{n}\left|v_{n}\right|^{p+1} x^{1} d x+\int_{\Omega_{n}-B_{R_{1}}\left(t_{n} / d_{n}\right)} Q_{n}\left|v_{n}\right|^{p+1} x^{1} d x \\
\geq & \left(\frac{t_{n}^{1}}{d_{n}}-R_{1}\right) \int_{\Omega_{n} \cap B_{R_{1}}\left(t_{n} / d_{n}\right)} Q_{n}\left|v_{n}\right|^{p+1} d x \\
& -\frac{|s|}{d_{n}} \int_{\Omega_{n}-B_{R_{1}}\left(t_{n} / d_{n}\right)} Q_{n}\left|v_{n}\right|^{p+1} d x \\
\geq & \left(\frac{t_{n}^{1}}{d_{n}}-R_{1}\right)(1-\varepsilon)-\frac{|s|}{d_{n}} \varepsilon
\end{aligned}
$$

So

$$
\beta\left(u_{n}\right)^{1} \geq\left(t_{n}^{1}-d_{n} R_{1}\right)(1-\varepsilon)-|s| \varepsilon .
$$

Letting $n \rightarrow \infty$ and $\varepsilon \rightarrow 0$ gives the contradiction $0 \geq t^{1}>0$.

We recall that the Lusternik-Schnirelman category of a subset $A \subset \partial \Omega$, denoted by $\operatorname{cat}_{\partial \Omega}(A)$, equals $k$ if $A$ can be covered by $k$ closed contractible sets in $\partial \Omega$, but not $k-1$ such sets. We set $\operatorname{cat}_{\partial \Omega}(\partial \Omega)=\operatorname{cat}(\partial \Omega)$.

Lemmas 6 and 7 below are proved exactly as in [14].

Lemma 6. Let $\varepsilon_{1}$ be given as in Proposition 3. For any $\varepsilon \in\left(0, \varepsilon_{1}\right)$ there exists $d_{\varepsilon}>0$ such that

$$
\operatorname{cat}\left(E_{d}^{c_{d}+\varepsilon_{d}}\right) \geq 2 \operatorname{cat}(\partial \Omega)
$$

where $\varepsilon_{d}=d^{N(p-1) /(p+1)} \varepsilon$.

LEMMA 7. Let $u$ be a critical point of $E_{d}(u)$ with

$$
E_{d}(u) \leq 2^{(p-1) /(p+1)} c_{d}
$$

Then $u$ does not change sign.

Now we prove the main result of this section.

THEOREM 3. If $d$ is sufficiently small, then $\left(1_{d}\right)$ has at least $\operatorname{cat}(\partial \Omega)$ distinct solutions.

Proof. From Proposition 3,

$$
c_{d}=d^{N(p-1) /(p+1)}\left(m\left(+, \frac{1}{M_{2}}\right)+o(1)\right)
$$

as $d \rightarrow 0$. For $\varepsilon_{1}$ as in Proposition 3 , choose $\varepsilon_{0} \in\left(0, \varepsilon_{1}\right]$ such that

$$
\varepsilon_{0}<\left(2^{(p-1) /(p+1)}-1\right) m\left(+, \frac{1}{M_{2}}\right) \text {. }
$$


Then there exists $d_{0}$ such that for all $d \in\left(0, d_{0}\right)$,

$$
c_{d}+d^{N(p-1) /(p+1)} c_{d}<2^{(p-1) /(p+1)} c_{d} .
$$

For this $\varepsilon_{0} \in\left(0, \varepsilon_{1}\right]$, there exists, by Lemma 6 , a $d_{0}^{\prime}>0$ such that

$$
\operatorname{cat}\left(E_{d}^{c_{d}+\varepsilon_{d}}\right) \geq 2 \operatorname{cat}(\partial \Omega)
$$

for all $d \in\left(0, d_{0}^{\prime}\right)$, where $\varepsilon_{d}=d^{N(p-1) /(p+1)} \varepsilon_{0}$.

Applying the minimax method here yields at least $2 \operatorname{cat}(\partial \Omega)$ critical points of $E_{d}$. From Lemma 7 none of the solutions change sign and so there are at least $\operatorname{cat}(\partial \Omega)$ solutions of $\left(1_{d}\right)$.

5. Multi-peak solutions. This section is devoted to the construction of multi-peak solutions. We aim to show that local minima of the restriction of $Q$ to $\partial \Omega$ generate multi-peak solutions. Our approach is a modification of the construction from [4].

For a fixed integer $k \geq 1$ we set $\alpha=\left(\alpha_{1}, \ldots, \alpha_{k}\right) \in \mathbb{R}^{k}, x=\left(x^{1}, \ldots, x^{k}\right)$ $\in \mathbb{R}^{k N}$, where $x^{i} \in \mathbb{R}^{N}, i=1, \ldots, k$. For $R>0$ we define

$$
D_{d, R}=\left\{x: x^{i} \in \partial \Omega, i=1, \ldots, k, \frac{\left|x^{i}-x^{j}\right|}{d} \geq R, i \neq j\right\} .
$$

We denote by $U$ the ground state solution of (3) with $M=1$. We use the notation $U_{d, z}(y)=U((y-z) / d)$. It is convenient for our purposes to define a scalar product in $H^{1}(\Omega)$ by

$$
\langle u, v\rangle_{d}=\int_{\Omega}\left(d^{2} \nabla u \nabla v+u v\right) d x
$$

and let $\|u\|_{d}=\langle u, u\rangle_{d}^{1 / 2}$. For every $x=\left(x^{1}, \ldots, x^{k}\right) \in \partial \Omega \times \ldots \times \partial \Omega$, we set

$$
\begin{aligned}
E_{d, x, k}=\left\{v \in H^{1}(\Omega):\left\langle U_{d, x^{i}}, v\right\rangle_{d}=\right. & \left\langle\frac{\partial U_{d, x^{i}}}{\partial \tau_{i, j}}, v\right\rangle_{d}=0, \\
& i=1, \ldots, k, j=1, \ldots, N-1\},
\end{aligned}
$$

where $\left\{\tau_{i, 1}, \ldots, \tau_{i, N-1}\right\}$ form an orthogonal basis of the tangent space to $\partial \Omega$ at $x^{i}$. Let $Q_{\circ}=\min _{x \in \partial \Omega} Q(x)$ and set

$$
\begin{aligned}
M_{d, \delta, R}=\left\{(\alpha, x, v):\left|\alpha_{i}-Q_{\circ}^{-1 /(p-1)}\right| \leq \delta,\right. & i=1, \ldots, k, \\
x \in D_{d, R}, v & \left.\in E_{d, x, k},\|v\|_{d} \leq \delta d^{N / 2}\right\}
\end{aligned}
$$

for some constant $\delta>0$. We define a functional $J_{d}: M_{d, \delta, R} \rightarrow \mathbb{R}$ by

$$
J_{d}(\alpha, x, v)=I_{d}\left(\sum_{i=1}^{k} \alpha_{i} U_{d, x^{i}}+v\right) .
$$

The proof of the following lemma is similar to that of Proposition 7 in [2]. 
LEMma 8. There exist constants $d_{\circ}>0, \delta>0$ and $R>0$ such that for every $d \in\left(0, d_{\circ}\right]$, every $u \in H^{1}(\Omega)$ satisfying

$$
\left\|u-\sum_{i=1}^{k} U_{d, x^{i}}\right\|_{d} \leq \delta d^{N / 2}
$$

for some $x=\left(x^{1}, \ldots, x^{k}\right) \in D_{d, R}$ admits a unique decomposition

$$
u=\sum_{i=1}^{k} \alpha_{d, i} U_{d, x_{d}^{i}}+v_{d},
$$

where $\left(\alpha_{d}, x_{d}, v_{d}\right) \in M_{d, \delta, k}$.

The next result is a consequence of Lemma 8 and shows that in order to find critical points of $I_{d}$ it is sufficient to find critical points of the functional $J_{d}$.

Proposition 5. There exist $d_{\circ}>0, \delta>0$ and $R>0$ such that for every $d \in\left(0, d_{\circ}\right]$, a point $(\alpha, x, v) \in M_{d, \delta, R}$ is a critical point of $J_{d}$ if and only if

$$
u=\sum_{i=1}^{k} \alpha_{i} U_{d, x^{i}}+v
$$

is a critical point of $I_{d}$.

It is known [13] that critical points of $I_{d}$ of the form $u=\sum_{i=1}^{k} \alpha_{i} U_{d, x^{i}}+v$ are positive. According to Proposition 4 to find a critical point $(\alpha, x, v) \in$ $M_{d, \delta, R}$ of $J_{d}$ it is enough to find $(\alpha, x, v) \in M_{d, \delta, R}$ and constants $A_{l}, B_{l i}$, $l=1, \ldots, k, i=1, \ldots, N-1$, such that

$$
\begin{aligned}
\frac{\partial J_{d}(\alpha, x, v)}{\partial \tau_{l, i}}=\sum_{j=1}^{N-1} B_{l j}\left\langle\frac{\partial^{2} U_{d, x^{l}}}{\partial \tau_{l, i} \partial \tau_{l, j}}, v\right\rangle_{d} & \\
& i=1, \ldots, N-1, l=1, \ldots, k,
\end{aligned}
$$

$$
\begin{aligned}
& \frac{\partial J_{d}(\alpha, x, v)}{\partial \alpha_{l}}=0, \quad l=1, \ldots, k \\
& \frac{\partial J_{d}(\alpha, x, v)}{\partial v}=\sum_{i=1}^{k} A_{i} U_{d, x^{i}}+\sum_{l=1}^{k} \sum_{j=1}^{N-1} B_{l j} \frac{\partial U_{d, x^{l}}}{\partial \tau_{l, j}} .
\end{aligned}
$$

In the first step we solve equations (25) and (26) for each fixed $x \in D_{d, R}$. Then we solve (24).

Proposition 6. There exist $d_{\circ}>0, \delta>0$ and $R>0$ such that for each $d \in\left(0, \delta_{\circ}\right]$ there exists a $C^{1}-\operatorname{map}\left(\alpha_{d}(x), v_{d}(x)\right): D_{d, R} \rightarrow \mathbb{R}^{k} \times E_{d, x, k}$ satisfying

$$
\frac{\partial J_{d}\left(\alpha_{d}(x), x, v_{d}(x)\right)}{\partial \alpha_{l}}=0, \quad l=1, \ldots, k
$$




$$
\left\langle\frac{\partial J_{d}\left(\alpha_{d}(x), x, v_{d}(x)\right)}{\partial v}, w\right\rangle_{d}=0
$$

for each $w \in E_{d, x, k}$, and moreover,

$$
\begin{aligned}
\left|\alpha_{l}-Q_{\circ}^{-1 /(p-1)}\right| & =O\left(d+\sum_{i \neq j} \exp \left(-\frac{1+\sigma}{2} \frac{\left|x^{i}-x^{j}\right|}{d}\right), \quad l=1, \ldots, k,\right. \\
\left\|v_{d}\right\|_{d} & =O\left(d^{N / 2}\left(d+\sum_{i \neq j} \exp \left(-\frac{1+\sigma}{2} \frac{\left|x^{i}-x^{j}\right|}{d}\right)\right)\right.
\end{aligned}
$$

for some constant $\sigma>0$.

Results of this nature are known and we refer to [4], [13]. Let $\left(\alpha_{d}, v_{d}\right)$ be the mapping from Proposition 6. Then there exists $x_{d} \in D_{d, R}$ such that

$$
J_{d}\left(\alpha_{d}\left(x_{d}\right), x_{d}, v_{d}\left(x_{d}\right)\right)=\sup \left\{J_{d}\left(\alpha_{d}(x), x, v_{d}(x)\right): x \in D_{d, R}\right\} .
$$

To proceed further we need some estimates of $J_{d}$. Let $H(x)$ be the mean curvature of $\partial \Omega$.

LEMMA 9. Let $x \in \partial \Omega$ and set

$$
K(x)=\frac{1}{2} H(x) \int_{\mathbb{R}^{N-1}} U^{p+1}\left(y^{\prime}, 0\right)\left|y^{\prime}\right|^{2} d y .
$$

Then

$$
\int_{\Omega} Q(y) U_{d, x}^{p+1}(y) d y=d^{N} Q(x)\left(A-d K(x)+O\left(d^{2}\right)\right)+O\left(d^{N+\alpha}\right),
$$

where $A=\frac{1}{2} \int_{\mathbb{R}^{N}} U^{p+1}(y) d y$ and $\alpha$ is a Hölder exponent for $Q$.

Proof. For simplicity we assume that $x=0$. We choose the coordinate system so that

$$
\Omega \cap B_{\tau}(0)=\left\{y_{N}>f\left(y^{\prime}\right)\right\}, \quad \partial \Omega \cap B_{\tau}(0)=\left\{y_{N}=f\left(y^{\prime}\right)\right\},
$$

where $\tau>0$ is a small constant and $f\left(y^{\prime}\right)$ satisfies

$$
f\left(y^{\prime}\right)=\frac{1}{2} \sum_{i=1}^{N-1} \varrho_{i} y_{i}^{2}+O\left(\left|y^{\prime}\right|^{3}\right) \quad \text { for } y^{\prime} \in B_{\tau}^{N-1}(0)=\left\{y^{\prime}:\left|y^{\prime}\right| \leq \tau\right\} .
$$

Then $H(0)=(N-1)^{-1} \sum_{i=1}^{N-1} \varrho_{i}$. Letting $\Omega_{d, x}=\{y: d y+x \in \Omega\}$, $\Omega_{d}=\Omega_{d, 0}$ and using the result from [4] (see also [8]) we get

$$
\begin{aligned}
\int_{\Omega} Q(y) U_{d, 0}^{p+1}(y) d y= & Q(0) \int_{\Omega} U_{d, 0}^{p+1}(y) d y+\int_{\Omega}(Q(y)-Q(0)) U_{d, 0}^{p+1}(y) d y \\
= & d^{N} Q(0)\left(A-d K(0)+O\left(d^{2}\right)\right) \\
& +\int_{\Omega}(Q(y)-Q(0)) U_{d, 0}^{p+1}(y) d y .
\end{aligned}
$$


It follows from the Hölder condition for $Q$ that

$$
\begin{aligned}
\left|\int_{\Omega}(Q(y)-Q(0)) U_{d, 0}^{p+1}(y) d y\right| & \leq C \int_{\Omega}|y|^{\alpha} U_{d, 0}^{p+1}(y) d y \\
& =C d^{N+\alpha} \int_{\Omega_{d}}|y|^{\alpha} U^{p+1}(y) d y \\
& \leq C d^{N+\alpha} \int_{\mathbb{R}^{N}}|y|^{\alpha} U^{p+1}(y) d y
\end{aligned}
$$

for some constant $C>0$ and the result follows.

We also need the following asymptotic relation (see Lemma A.2 in [4]):

$$
\int_{\Omega}\left(d^{2}\left|\nabla U_{d, x}\right|^{2}+U_{d, x}^{2}\right) d x=d^{N}\left(A-d K(x)+d M(x)+O\left(d^{2}\right)\right),
$$

where $K(x)$ is as in Lemma 9 and

$$
M(x)=\frac{1}{2} H(x) \int_{\mathbb{R}^{N-1}} U\left(y^{\prime}, 0\right) \frac{\partial U\left(y^{\prime}, 0\right)}{\partial r}\left|y^{\prime}\right| d y .
$$

Proposition 7. Let $x_{d}$ be a point in $D_{d, R}$ where $J_{d}$ attains its maximum. Then

$$
\frac{\left|x_{d}^{i}-x_{d}^{j}\right|}{d} \rightarrow \infty \quad \text { as } d \rightarrow 0, i \neq j
$$

$x_{d}^{i} \rightarrow x^{i} \in \partial \Omega$ as $d \rightarrow 0$ and $x^{i}$ satisfies $Q\left(x^{i}\right)=\min _{\partial \Omega} Q(x), i=1, \ldots, k$.

Proof. Expanding $J_{d}(\alpha, x, v)$ around $(\bar{Q}, x, 0)$, where $\bar{Q}=\left(Q_{\circ}^{-1 /(p-1)}\right.$, $\left.\ldots, Q_{\circ}^{-1 /(p-1)}\right)$ and using Proposition 6 we get

$$
\begin{aligned}
& J_{d}\left(\alpha_{d}(x), x, v_{d}(x)\right) \\
& \quad=J_{d}(\bar{Q}, x, 0)+O\left(d^{N}\left(d^{2}+\sum_{i \neq j} \exp \left(-(1+\sigma) \frac{\left|x^{i}-x^{j}\right|}{d}\right)\right)\right) .
\end{aligned}
$$

Since $x_{d}$ is a point in $D_{d, R}$ where the maximum is achieved we have

$$
J_{d}\left(\alpha_{d}\left(x_{d}\right), x_{d}, v_{d}\left(x_{d}\right)\right) \geq J_{d}\left(\alpha_{d}\left(z_{d}\right), z_{d}, v_{d}\left(z_{d}\right)\right)
$$

for every $z_{d} \in D_{d, R}$. Thus

$$
\begin{aligned}
& J_{d}\left(\bar{Q}, x_{d}, 0\right)+O\left(d^{N}\left(d^{2}+\sum_{i \neq j} \exp \left(-(1+\sigma) \frac{\left|x_{d}^{i}-x_{d}^{j}\right|}{d}\right)\right)\right) \\
& \geq J_{d}\left(\bar{Q}, z_{d}, 0\right)+O\left(d^{N}\left(d^{2}+\sum_{i \neq j} \exp \left(-(1+\sigma) \frac{\left|z_{d}^{i}-z_{d}^{j}\right|}{d}\right)\right)\right) .
\end{aligned}
$$

Let $e_{i}, i=1, \ldots, k$, be tangent vectors to $\partial \Omega$ at $x_{\circ}$, with $e_{i} \neq e_{j}$ for $i \neq j$, and $z_{i}(t)$ be a curve in $\partial \Omega$ satisfying $z_{i}(0)=x_{\circ}, z_{i}^{\prime}(0)=e_{i}$, where $Q\left(x_{\circ}\right)=Q_{\circ}$. Let $z_{d}^{i}=z^{i}\left(d^{1 / 2}\right), i=1, \ldots, k$. Then 


$$
\frac{\left|z_{d}^{i}-z_{d}^{j}\right|}{d}=\frac{\left|e_{i}-e_{j}\right|+o(1)}{d^{1 / 2}} \rightarrow \infty \quad \text { as } d \rightarrow 0 .
$$

Therefore $z_{d} \in D_{d, R}$ if $d>0$ is sufficiently small. It follows from Lemma 9 and (29) that

$$
\begin{aligned}
& J_{d}\left(\bar{Q}, z_{d}, 0\right) \\
= & \frac{1}{2}\left\|\sum_{i=1}^{k} Q_{\circ}^{-1 /(p-1)} U_{d, z_{d}^{i}}\right\|_{d}^{2}-\frac{1}{p+1} \int_{\Omega} Q(y)\left|\sum_{i=1}^{k} Q_{\circ}^{-1 /(p-1)} U_{d, z_{d}^{i}}\right|^{p+1} d y \\
= & \frac{1}{2} \sum_{i=1}^{k} Q_{\circ}^{-2 /(p-1)}\left\|U_{d, z_{d}^{i}}\right\|_{d}^{2} \\
& -\frac{1}{p+1} \sum_{i=1}^{k} Q_{\circ}^{-(p+1) /(p-1)} \int_{\Omega} Q(y) U_{d, z_{d}^{i}}^{p+1} d y+O\left(d^{N} e^{-c_{\circ} / d^{1 / 2}}\right) \\
= & \frac{d^{N}}{2}\left(\sum_{i=1}^{k} Q_{\circ}^{-2 /(p-1)}\left(A-d K\left(z_{d}^{i}\right)+d M\left(z_{d}^{i}\right)+O\left(d^{2}\right)\right)\right) \\
& -\frac{d^{N}}{p+1} \sum_{i=1}^{k} Q\left(z_{d}^{i}\right) Q_{\circ}^{-(p+1) /(p-1)}\left(A-d K\left(z_{d}^{i}\right)+O\left(d^{2}\right)\right)+O\left(d^{N+\alpha}\right) \\
= & \frac{k d^{N}}{2}\left(Q_{\circ}^{-2 /(p-1)}\left(A-d K\left(x_{\circ}\right)+d M\left(x_{\circ}\right)+O\left(d^{3 / 2}\right)\right)\right) \\
& -\frac{k d^{N}}{p+1} Q_{\circ}^{-2 / p-1}\left(A-d K\left(x_{\circ}\right)+O\left(d^{3 / 2}\right)\right)+O\left(d^{N+\alpha}\right) \\
= & k\left(\frac{1}{2}-\frac{1}{p+1}\right) d^{N} A Q_{\circ}^{-2 /(p-1)}+O\left(d^{N+\alpha}\right)
\end{aligned}
$$

for some constant $c_{\circ}>0$. For $J_{d}\left(\bar{Q}, x_{d}, 0\right)$ we have the estimate

$$
\begin{aligned}
J_{d}\left(\bar{Q}, x_{d}, 0\right)= & \frac{1}{2}\left\|\sum_{i=1}^{k} Q_{\circ}^{-1 /(p-1)} U_{d, x_{d}^{i}}\right\|_{d}^{2} \\
& -\frac{1}{p+1} \int_{\Omega} Q(y)\left|\sum_{i=1}^{k} Q_{\circ}^{-1 /(p-1)} U_{d, x_{d}^{i}}\right|^{p+1} d y \\
= & \sum_{i=1}^{k}\left(\frac{1}{2} Q_{\circ}^{-2 /(p-1)}\left\|U_{d, x_{d}^{i}}\right\|_{d}^{2}\right. \\
& \left.-\frac{1}{p+1} Q_{\circ}^{-(p+1) /(p-1)} \int_{\Omega} Q(y) U_{d, x_{d}^{i}}^{p+1} d y\right)
\end{aligned}
$$




$$
\begin{aligned}
& +\frac{1}{2} \sum_{i \neq j} Q_{\circ}^{-2 /(p-1)}\left\langle U_{d, x_{d}^{i}}, U_{d, x_{d}^{j}}\right\rangle_{d} \\
& -\frac{1}{p+1}\left[\int_{\Omega} Q(y)\left|\sum_{i=1}^{k} Q_{\circ}^{-1 /(p-1)} U_{d, x_{d}^{i}}\right|^{p+1} d y\right. \\
& \left.-\sum_{i=1}^{k} \int_{\Omega} Q_{\circ}^{-(p+1) /(p-1)} Q(y) U_{d, x_{d}^{i}}^{p+1} d y\right] .
\end{aligned}
$$

We denote the last two terms on the right side by $P_{1}$ and $-\frac{1}{p+1} P_{2}$, respectively. For $P_{1}$ we have the estimate (see [4])

$$
\begin{aligned}
P_{1}= & \frac{1}{2} \sum_{i \neq j} Q_{\circ}^{-2 /(p-1)}\left\langle U_{d, x_{d}^{i}}, U_{d, x_{d}^{j}}\right\rangle_{d} \\
= & \sum_{i<j} Q_{\circ}^{-2 /(p-1)} \int_{\Omega} U_{d, x_{d}^{i}}^{p} U_{d, x_{d}^{j}} d y \\
& +O\left(d^{N+1} \sum_{i \neq j} \exp \left(-\frac{(1-\theta)\left|x_{d}^{i}-x_{d}^{j}\right|}{d}\right)\right) .
\end{aligned}
$$

To estimate $P_{2}$ we make repeated use of the inequality

$$
\begin{aligned}
|| a+\left.b\right|^{p}-a^{p}-b^{p}-p a^{p-1} b- & p a b^{p-1} \mid \\
& \leq \begin{cases}C a^{p / 2} b^{p / 2} & \text { if } 2<p \leq 3, \\
C\left(a^{p-2} b^{2}+a^{2} b^{p-2}\right) & \text { if } p>3,\end{cases}
\end{aligned}
$$

and we get

$$
\begin{aligned}
P_{2}= & \int_{\Omega} Q(y)\left|\sum_{i=1}^{k} Q_{\circ}^{-1 /(p-1)} U_{d, x_{d}^{i}}\right|^{p+1} d y-\sum_{i=1}^{k} \int_{\Omega} Q_{\circ}^{-(p+1) /(p-1)} Q(y) U_{d, x_{d}^{i}}^{p+1} d y \\
= & Q_{\circ}^{-(p+1) /(p-1)} \int_{\Omega} Q(y)\left(\sum_{i=2}^{k} U_{d, x_{d}^{i}}\right)^{p+1} d y \\
& -Q_{\circ}^{-(p+1) /(p-1)} \sum_{i=2}^{k} \int_{\Omega} Q(y) U_{d, x_{d}^{i}}^{p+1} d y \\
& +(p+1) Q_{\circ}^{-(p+1) /(p-1)} \int_{\Omega} Q(y)\left(\sum_{i=2}^{k} U_{d, x_{d}^{i}}\right)^{p} U_{d, x_{d}^{1}} d y \\
& +(p+1) Q_{\circ}^{-(p+1) /(p-1)} \int_{\Omega} Q(y) U_{d, x_{d}^{1}}^{p} \sum_{i=2}^{k} U_{d, x_{d}^{i}} d y \\
& +O\left(d^{N} \sum_{i \neq j} \exp \left(-\frac{(1+\sigma)\left|x_{d}^{i}-x_{d}^{j}\right|}{d}\right)\right)
\end{aligned}
$$




$$
\begin{aligned}
= & (p+1) Q_{\circ}^{-(p+1) /(p-1)} \int_{\Omega} Q(y) \sum_{j=1}^{k-1}\left(\sum_{i=j+1}^{k} U_{d, x_{d}^{i}}\right)^{p} U_{d, x_{d}^{j}} d y \\
& +(p+1) Q_{\circ}^{-(p+1) /(p-1)} \int_{\Omega} Q(y) \sum_{i<j} U_{d, x_{d}^{i}}^{p} U_{d, x_{d}^{j}} d y \\
& +O\left(d^{N} \sum_{i \neq j} \exp \left(-\frac{(1+\sigma)\left|x_{d}^{i}-x_{d}^{j}\right|}{d}\right)\right) .
\end{aligned}
$$

Since

$$
\begin{gathered}
Q_{\circ}^{-2 /(p-1)} \sum_{i<j} \int_{\Omega} U_{d, x_{d}^{i}}^{p} U_{d, x_{d}^{j}} d y-Q_{\circ}^{-(p+1) /(p-1)} \int_{\Omega} Q(y) \sum_{i<j} U_{d, x_{d}^{i}}^{p} U_{d, x_{d}^{j}} d y \\
=Q_{\circ}^{-2 /(p-1)} \int_{\Omega}\left(1-\frac{Q(y)}{Q_{\circ}}\right) \sum_{i<j} U_{d, x_{d}^{i}}^{p} U_{d, x_{d}^{j}} d y<0,
\end{gathered}
$$

we derive the following estimate:

$(32)$

$$
\begin{aligned}
J_{d}\left(\bar{Q}, x_{d}, 0\right) & \\
\leq & d^{N}\left[\frac{k}{2} A \bar{Q}_{\circ}^{-2 /(p-2)}-\frac{1}{p+1} A \sum_{i=1}^{k} Q_{\circ}^{-(p+1) /(p-1)} Q\left(x_{d}^{i}\right)\right. \\
& \left.-\left(\frac{1}{2}-\frac{1}{p+1}\right) d \sum_{i=1}^{k} Q_{\circ}^{-2 /(p-1)} K\left(x_{d}^{i}\right)+O\left(d^{2}\right)\right]+O\left(d^{N+\alpha}\right) \\
& -Q_{\circ}^{-(p+1) /(p-1)} \int_{\Omega} Q(y) \sum_{j=1}^{k-1}\left(\sum_{i=j+1}^{k} U_{d, x_{d}^{i}}\right)^{p} U_{d, x_{d}^{j}} d y \\
& +O\left(d^{N} \sum_{i \neq j} \exp \left(-\frac{\left.(1+\sigma)\left|x_{d}^{i}-x_{d}^{j}\right|\right)}{d}\right)\right) .
\end{aligned}
$$

Inserting estimates (31) and (32) into (30) we get

$$
\begin{aligned}
& -\frac{k}{p+1} A Q_{\circ}^{-2 /(p-1)} \\
& \leq-\frac{1}{p+1} A Q_{\circ}^{-(p+1) /(p-1)} \sum_{i=1}^{k} Q\left(x_{d}^{i}\right) \\
& \quad-d^{-N} Q_{\circ}^{-(p+1) /(p-1)} \int_{\Omega} Q(y) \sum_{j=1}^{k-1}\left(\sum_{i=j+1}^{k} U_{d, x_{d}^{i}}\right)^{p} U_{d, x_{d}^{j}} d y \\
& \quad+O\left(\sum_{i \neq j} \exp \left(\frac{(1+\sigma)\left|x_{d}^{i}-x_{d}^{j}\right|}{d}\right)\right)+O\left(d^{\alpha}\right) .
\end{aligned}
$$


Since

$$
\begin{aligned}
d^{-N} Q_{\circ}^{-(p+1) /(p-1)} \int_{\Omega} Q(y) \sum_{j=1}^{k-1}\left(\sum_{i=j+1}^{k} U_{d, x_{d}^{i}}\right)^{p} U_{d, x_{d}^{j}} d y \\
\quad \geq c_{\circ} \sum_{i \neq j} \exp \left(-\frac{(1+\sigma / 2)\left|x_{d}^{i}-x_{d}^{j}\right|}{d}\right)
\end{aligned}
$$

for some $c_{\circ}>0$ and for large $R>0$ we deduce from (33) that

$$
\begin{aligned}
\sum_{i=1}^{k} Q\left(x_{d}^{i}\right)+O\left(d^{\alpha}\right)+c_{\circ} & \sum_{i \neq j} \exp \left(-\left(1+\frac{\sigma}{2}\right) \frac{\left|x_{d}^{i}-x_{j}^{d}\right|}{d}\right) \\
& +O\left(\sum_{i \neq j} \exp \left(-(1+\sigma) \frac{\left|x_{d}^{i}-x_{d}^{j}\right|}{d}\right)\right) \leq k Q_{\circ} .
\end{aligned}
$$

Taking $R$ sufficiently large we deduce that

$$
\sum_{i=1}^{k} Q\left(x_{d}^{i}\right)+O\left(d^{\alpha}\right)+c_{1} \sum_{i \neq j} \exp \left(-\left(1+\frac{\sigma}{2}\right) \frac{\left|x_{i}^{i}-x_{d}^{j}\right|}{d}\right) \leq k Q_{\circ} .
$$

Since $Q\left(x_{d}^{i}\right) \geq Q_{\circ}, i=1, \ldots, k$, we see that

$$
\lim _{d \rightarrow 0} \frac{\left|x_{d}^{i}-x_{d}^{j}\right|}{d}=\infty
$$

and necessarily $x_{d}^{i} \rightarrow x_{i}$ as $d \rightarrow 0$. Letting $d \rightarrow 0$ in (34) we get

$$
\sum_{i=1}^{k} Q\left(x_{i}\right) \leq k Q\left(x_{\circ}\right)=k \min _{x \in \partial \Omega} Q(x)
$$

and the result follows.

From Propositions 6 and 7 we deduce the following existence result:

THEOREM 4. For each positive integer $k$, there exists $a d_{\circ}=d_{\circ}(k)$ such that for each $d \in\left(0, d_{\circ}\right]$, problem $\left(1_{d}\right)$ has a solution of the form

$$
u_{d}=\sum_{i=1}^{k} \alpha_{d}^{i} U_{d, x_{d}^{i}}+v_{d}
$$

where

$$
\begin{gathered}
\alpha_{d}^{i} \rightarrow Q_{i}^{-1 /(p-1)}, \quad i=1, \ldots, k, \\
\frac{\left|x_{d}^{i}-x_{d}^{j}\right|}{d} \rightarrow \infty \quad \text { for } i \neq j, \quad x_{d}^{i} \rightarrow x^{i}
\end{gathered}
$$

as $d \rightarrow 0$ and $x_{d}^{i}$ satisfies $Q\left(x^{i}\right)=\min _{\partial \Omega} Q(x), k=1, \ldots, l$. In particular, if $\left.Q\right|_{\partial \Omega}$ has only one global minimum point at $x_{\circ}$, then $x_{d}^{i} \rightarrow x_{\circ}$ as $d \rightarrow 0$, $i=1, \ldots, k$. 
6. Effect of the graph topology of the coefficient $Q$. In this section we examine the effect of the graph topology of the coefficient $Q$ on the number of one-peak solutions. We follow some ideas from the paper [3], where the effect of the graph topology of $Q$ was studied for the Dirichlet problem. Let $Q_{\mathrm{m}}=\max _{x \in \partial \Omega} Q(x)$ and set

$$
\mathcal{M}=\left\{x \in \partial \Omega: Q(x)=Q_{\mathrm{m}}\right\} \quad \text { and } \quad \mathcal{M}_{\varrho}=\{x \in \partial \Omega: \operatorname{dist}(x, \mathcal{M})<\varrho\},
$$

where $\varrho>0$ is a small number. The sets $E_{d, x, k}$, with $k=1$, introduced in Section 5 , are now denoted by $E_{d, x}$, that is,

$$
E_{d, x}=\left\{v \in H^{1}(\Omega):\left\langle U_{d, x}, v\right\rangle_{d}=\left\langle\frac{\partial U_{d, x}}{\partial \tau_{i}}, v\right\rangle_{d}=0, i=1, \ldots, N-1\right\},
$$

where $\left\{\tau_{i}\right\}, i=1, \ldots, N-1$, is an orthogonal basis of the tangent space to $\partial \Omega$ at $x$. For each $\delta>0$ small enough we define

$\mathcal{M}_{d, \varrho}=\left\{(\alpha, x, v):\left|\alpha-Q_{\mathrm{m}}^{-1 /(p-1)}\right| \leq \delta, x \in \mathcal{M}_{\varrho}, v \in E_{d, x},\|v\|_{d} \leq \delta d^{N / 2}\right\}$.

For $(\alpha, x, v) \in \mathcal{M}_{d, \varrho}$ we set

$$
J_{d}(\alpha, x, v)=I_{d}\left(\alpha U_{d, x}+v\right) .
$$

As in Section 5 we have the following result:

Proposition 8. There exist $d_{\circ}>0, \delta>0$ such that for every $d \in\left(0, d_{\circ}\right)$ a point $(\alpha, x, v) \in \mathcal{M}_{d, \varrho}$ is a critical point of the functional $J_{d}(\alpha, x, v)$ if and only if $\alpha U_{d, x}+v$ is a critical point of $I_{d}$.

Consequently, to find a critical point $(\alpha, x, v) \in \mathcal{M}_{d, \varrho}$ we need to solve the following problem: find constants $A, B_{i}, i=1, \ldots, N-1$, and $(\alpha, x, v) \in$ $\mathcal{M}_{d, \varrho}$ such that

$$
\begin{aligned}
\frac{\partial J_{d}}{\partial \tau_{i}} & =\sum_{j=1}^{N-1} B_{j}\left\langle\frac{\partial^{2} U_{d, x}}{\partial \tau_{i} \partial \tau_{j}}, v\right\rangle_{d}, \quad i=1, \ldots, N-1, \\
\frac{\partial J_{d}}{\partial \alpha} & =0, \\
\frac{\partial J_{d}}{\partial v} & =A U_{d, x}+\sum_{j=1}^{N-1} B_{j} \frac{\partial U_{d, x}}{\partial \tau_{j}} .
\end{aligned}
$$

We need a result analogous to Proposition 6 .

Proposition 9. There exist $d_{\circ}>0, \delta>0$ such that for every $d \in$ $\left(0, d_{\circ}\right)$, there exists a $C^{1}$-mapping $\left(\alpha_{d}(x), v_{d}(x)\right): \mathcal{M}_{\varrho} \rightarrow \mathbb{R} \times E_{d, x}$ satisfying

$$
\frac{\partial J_{d}\left(\alpha_{d}(x), x, v_{d}(x)\right)}{\partial \alpha}=0, \quad\left\langle\frac{\partial J_{d}\left(\alpha_{d}(x), x, v_{d}(x)\right)}{\partial v}, w\right\rangle_{d}=0
$$


for every $w \in E_{d, x}$ and

$$
\left|\alpha_{d}(x)-Q_{\mathrm{m}}^{-1 /(p-1)}\right|=O\left(d^{N / 2+\alpha}\right), \quad\left\|v_{d}\right\|_{d}=O\left(d^{N / 2+1}\right) .
$$

Proof. Let $J^{*}(x, w)=J(\alpha, x, v)=I_{d}\left(\alpha U_{d, x}+v\right)$, where $w=(\beta, v)=$ $\left(\alpha-Q_{\mathrm{m}}^{-1 /(p-d)}, v\right)$. As in [4] (see also [13]) we expand $J^{*}(x, w)$ at $w=0$ :

$$
J^{*}(w, x)=J^{*}(0, x)+\left\langle f_{d, x}, w\right\rangle_{d}+\frac{1}{2}\left\langle Q_{d, x} w, w\right\rangle+R_{d, x}(w),
$$

where $f_{d, x} \in \mathbb{R} \times E_{d, x}$ is given by

$$
\begin{aligned}
\left\langle f_{d, x}, w\right\rangle_{d}= & {\left[Q_{m}^{-1 /(p-1)}\left\|U_{d, x}\right\|_{d}^{2}-Q_{\mathrm{m}}^{-p /(p-1)} \int_{\Omega} Q(y) U_{d, x}^{p+1} d y\right] \beta } \\
& -Q_{\mathrm{m}}^{-p /(p-1)} \int_{\Omega} Q(y) U_{d, x}^{p} v d y \\
= & J_{1} \cdot \beta+J_{2}(v),
\end{aligned}
$$

$Q_{d, x}$ is a linear map from $\mathbb{R} \times E_{d, x}$ to $\mathbb{R} \times E_{d, x}$ and $R_{d, x}$ is the higher order term satisfying

$$
R_{d, x}^{(i)}(w)=O\left(\|w\|_{d}^{\min (p-i, 3-i)}\right), \quad i=0,1,2 .
$$

Repeating the argument from [4] we show that $Q_{d, x}$ is invertible and $\left\|Q_{d, x}^{-1}\right\|$ $\leq C$, where $C$ is independent of $d$ and $x$. Obviously, equations (36) and (37) are equivalent to

$$
f_{d, x}+Q_{d, x} w+R_{d, x}^{\prime}(w)=0 .
$$

From the implicit function theorem this equation has a solution $w_{d} \in \mathbb{R} \times$ $E_{d, x}$ and $w_{d}$ satisfies

$$
\left\|w_{d}\right\|_{d} \leq C\left\|f_{d, x}\right\| .
$$

We now estimate $\left\|f_{d, x}\right\|$. For the term $J_{1}$ of $\beta$ we have (we eventually drop the $d, x$ subscript)

$$
\begin{aligned}
J_{1}= & Q_{\mathrm{m}}^{-1 /(p-1)}\left\|U_{d, x}\right\|_{d}^{2}-Q_{\mathrm{m}}^{-p /(p-1)} \int_{\Omega} Q(y) U_{d, x}^{p+1} d y \\
= & Q_{\mathrm{m}}^{-1 /(p-1)}\left[\int_{\Omega}\left(d^{2}|\nabla U|^{2}+U^{2}\right) d y-Q_{\mathrm{m}}^{-1} \int_{\Omega} Q(y) U^{p+1} d y\right] \\
= & Q_{\mathrm{m}}^{-1 /(p-1)}\left[\int_{\Omega}\left(U^{2}-\frac{Q(y)}{Q_{\mathrm{m}}} U^{p+1}\right) d y+\int_{\Omega} d^{2}|\nabla U|^{2} d y\right] \\
= & Q_{\mathrm{m}}^{-1 /(p-1)}\left[\int_{\Omega}\left(U^{2}-\frac{Q(y)}{Q_{\mathrm{m}}} U^{p+1}\right) d y\right. \\
& \left.+\int_{\partial \Omega} d^{2} U \frac{\partial U}{\partial \nu} d \sigma-\int_{\Omega} d^{2} U \Delta U d y\right]
\end{aligned}
$$




$$
\begin{aligned}
= & Q_{\mathrm{m}}^{-1 /(p-1)}\left[\int_{\Omega}\left(-d^{2} \Delta U_{d, x}+U_{d, x}-U_{d, x}^{p}\right) d y\right. \\
& \left.+\int_{\Omega}\left(1-\frac{Q(y)}{Q_{\mathrm{m}}}\right) U_{d, x}^{p+1} d y+\int_{\partial \Omega} d^{2} U \frac{\partial U}{\partial \nu} d \sigma\right] .
\end{aligned}
$$

After scaling $z=(y-x) / d$ we find the first integral to be 0 . The third integral is $O\left(d^{N+1}\right)$. For the second integral we have the estimate

$$
\begin{aligned}
\int_{\Omega}\left(1-\frac{Q(y)}{Q_{\mathrm{m}}}\right) U_{d, x}^{p+1} d y= & d^{N}\left(A-d K(x)+O\left(d^{2}\right)\right) \\
& -d^{N} \frac{Q(x)}{Q_{\mathrm{m}}}\left(A-d K(x)+O\left(d^{2}\right)\right)+O\left(d^{N+\alpha}\right) \\
= & d^{N} A\left(1-\frac{Q(x)}{Q_{\mathrm{m}}}\right)+O\left(d^{N+\alpha}\right)+o\left(d^{N+1}\right) .
\end{aligned}
$$

The estimates for $\alpha_{d}$ and $\left\|v_{d}\right\|_{d}$ easily follow.

Problem $(1)_{d}$ is reduced to finding $x \in \mathcal{M}_{\varrho}$ such that equation (34) is satisfied.

Proposition 10. Let $\left(\alpha_{d}, v_{d}\right)$ be the mapping from Proposition 9. Then

$$
J_{d}\left(\alpha_{d}(x), x, v_{d}(x)\right)=d^{N}\left[\frac{A}{2 Q_{\mathrm{m}}^{2 /(p-1)}}\left(1-\frac{2}{p+1} \frac{Q(x)}{Q_{\mathrm{m}}}\right)+O(d)\right],
$$

where $A$ is the constant defined in Lemma 9.

Proof. It follows from Proposition 9 that

$$
\begin{aligned}
\int_{\Omega} d^{2}\left|\alpha_{d} \nabla U_{d, x}+\nabla v_{d}\right|^{2} d x & +\int_{\Omega}\left(\alpha_{d} U_{d, x}+v_{d}\right)^{2} d x \\
& =\alpha_{d}^{2}\left\|U_{d, x}\right\|_{d}^{2}+\left\|v_{d}\right\|_{d}^{2}+\left\langle U_{d, x}, v\right\rangle=\alpha_{d}^{2}\left\|U_{d, x}\right\|_{d}^{2}+O\left(d^{N+2}\right) .
\end{aligned}
$$

Next we have

$$
\begin{aligned}
& \int_{\Omega} Q(y)\left|\alpha_{d} U_{d, x}+v_{d}\right|^{p+1} d y \\
& =\int_{\Omega} Q(y)\left|\alpha_{d} U_{d, x}\right|^{p+1} d y \\
& \quad+(p+1) \int_{\Omega} Q(y)\left|\alpha_{d} U_{d, x}+\theta(y) v_{d}\right|^{p-1}\left(\alpha_{d} U_{d, x}+\theta(y) v_{d}\right) d y
\end{aligned}
$$

where $0<\theta \leq 1$. Hence 


$$
\begin{aligned}
\int_{\Omega} Q(y) \mid \alpha_{d} U_{d, x} & +\left.\theta(y) v_{d}\right|^{p}\left|v_{d}\right| d y \\
\leq & 2^{p} \int_{\Omega} Q(y)\left|\alpha_{d} U_{d, x}\right|^{p}\left|v_{d}\right| d y+2^{p} \int_{\Omega} Q(y)\left|v_{d}\right|^{p+1} d y \\
\leq & 2^{p} \max _{\bar{\Omega}} Q\left(\int_{\Omega}\left|\alpha_{d} U_{d, x}\right|^{p+1} d y\right)^{p /(p+1)}\left(\int_{\Omega}\left|v_{d}\right|^{p+1} d y\right)^{1 /(p+1)} \\
& +2^{p} \int_{\Omega}\left|v_{d}\right|^{p+1} d x \\
= & O\left(d^{N p /(p+1)+N / 2+1}\right)+O\left(d^{(N / 2+1)(p+1)}\right) .
\end{aligned}
$$

Inserting the above estimates into $I_{d}\left(\alpha_{d} U_{d, x}+v_{d}\right)$ we get

$$
\begin{aligned}
I_{d}\left(\alpha_{d} U_{d, x}+v_{d}\right)= & \frac{\alpha_{d}^{2}}{2}\left\|U_{d, x}\right\|_{2}^{2}-\frac{1}{p+1} \int_{\Omega} Q(y)\left|\alpha_{d} U_{d, x}\right|^{p+1} d y \\
& +O\left(d^{N+2}\right)+\left(O\left(d^{N+\alpha}\right)+O\left(d^{N}\right)\right) O\left(d^{N / 2+1}\right) \\
= & \frac{\alpha_{d}^{2}}{2}\left\|U_{d, x}\right\|_{2}^{2}-\frac{1}{p+1} \int_{\Omega} Q(y)\left|\alpha_{d} U_{d, x}\right|^{p+1} d y+O\left(d^{N+1}\right) .
\end{aligned}
$$

Applying Lemma 9 and the estimate (29) we obtain

$$
\begin{aligned}
I_{d}\left(\alpha_{d} U_{d, x}+v\right)= & \frac{\alpha_{d}^{2}}{2}\left[d^{N}\left(A-d K(x)+d M(x)+O\left(d^{2}\right)\right)\right] \\
& -\frac{\alpha_{d}^{p+1}}{p+1}\left[d^{N} Q(x)\left(A-d K(x)+O\left(d^{2}\right)\right)\right] \\
& +O\left(d^{N+\alpha}\right)+O\left(d^{N+1}\right) \\
= & \frac{\alpha_{d}^{2}}{2} d^{N} A\left(1-\frac{2 \alpha_{d}^{p-1}}{p+1} Q(x)\right) \\
& +\frac{\alpha_{d}^{2} d^{N}}{2}\left(d(M(x)-K(x))+O\left(d^{2}\right)\right) \\
& +\frac{\alpha_{d}^{p+1}}{p+1} d^{N} Q(x)\left(d K(x)+O\left(d^{2}\right)\right)+O\left(d^{N+\alpha}\right)+O\left(d^{N+1}\right) \\
= & \frac{\alpha_{d}^{2}}{2} d^{N} A\left(1-\frac{2 \alpha_{d}^{p-1}}{p+1} Q(x)\right)+O\left(d^{N+1}\right) .
\end{aligned}
$$

The result follows by applying the estimate $\left|\alpha_{d}-Q_{\mathrm{m}}^{-1 /(p-1)}\right|=O\left(d^{N / 2+\alpha}\right)$ from Proposition 9.

To proceed further we define a functional $F: \mathcal{M}_{\varrho} \rightarrow \mathbb{R}$ by $F(x)=$ $J_{d}\left(\alpha_{d}(x), x, v_{d}(x)\right)$, where $\left(\alpha_{d}, v_{d}\right)$ is the mapping from Proposition 9. Let 


$$
a_{d}=d^{N}\left[\frac{A}{2 Q_{\mathrm{m}}^{2 /(p-1)}}\left(1-\frac{2}{p+1}\right)+d^{s}\right],
$$

where $0<s<1$. We show that there exists $d_{1}>0$ sufficiently small such that the flow defined by

$$
\begin{gathered}
\frac{d Y(t)}{d t}=-\operatorname{grad} F(Y(t)), \\
Y_{\circ}=Y(0) \in F^{a_{d}}=\left\{x \in \mathcal{M}_{\varrho}: F(x)<a_{d}\right\}
\end{gathered}
$$

does not leave $\mathcal{M}_{\varrho}$ for $0<d \leq d_{1}$.

Lemma 10. There exists $d_{1}>0$ such that for every $d \in\left(0, d_{1}\right)$ and every $x \in \partial \mathcal{M}_{\varrho}$ we have $F(x)>a_{d}$.

Proof. Arguing by contradiction assume that there are sequences $d_{n} \rightarrow 0$ and $\left\{x_{n}\right\} \subset \partial M_{\varrho}$ such that $F\left(x_{n}\right) \leq a_{d_{n}}$. We then have

$$
\frac{A}{2 Q_{\mathrm{m}}^{2 /(p-1)}}\left(1-\frac{2}{p+1} \frac{Q\left(x_{n}\right)}{Q_{\mathrm{m}}}\right)+O\left(d_{n}\right) \leq \frac{A}{2 Q_{\mathrm{m}}^{2 /(p-1)}}\left(1-\frac{2}{p+1}\right)+d_{n}^{s} .
$$

We may assume that $x_{n} \rightarrow x_{\circ} \in \partial \mathcal{M}_{\varrho}$. Letting $n \rightarrow 0$ in the last inequality we deduce that $Q\left(x_{\circ}\right) / Q_{\mathrm{m}} \geq 1$, which contradicts the fact that $x_{\circ} \in \partial \mathcal{M}_{\varrho}$ and $Q\left(x_{\circ}\right)<Q_{\mathrm{m}}$.

Lemma 11. There exists $d_{2}>0$ small enough such that $\mathcal{M} \subset F^{a_{d}}$ for $0<d \leq d_{2}$.

Proof. In the contrary case there exist sequences $d_{n} \rightarrow 0$ and $\left\{x_{n}\right\} \subset \mathcal{M}$ such that $F\left(x_{n}\right)>a_{d_{n}}$. From this we get

$$
\begin{aligned}
d_{n}^{N}\left[\frac{A}{2 Q_{\mathrm{m}}^{2 /(p-1)}}\left(1-\frac{2}{p+1} \frac{Q\left(x_{n}\right)}{Q_{\mathrm{m}}}\right)\right. & \left.+O\left(d_{n}\right)\right] \\
& >d_{n}^{N}\left[\frac{A}{2 Q_{\mathrm{m}}^{2 /(p-1)}}\left(1-\frac{2}{p+1}\right)+d_{n}^{s}\right],
\end{aligned}
$$

which is impossible since $Q\left(x_{n}\right)=Q_{\mathrm{m}}$ and $0<s<1$.

Theorem 4. Suppose that $Q(x) \not \equiv$ Const on $\partial \Omega$. Then there exists a constant $\varrho>0$ such that problem $\left(1_{d}\right)$ has cat $_{\mathcal{M}_{\varrho}}(\mathcal{M})$ solutions for $0<d \leq$ $\min \left(d_{1}, d_{2}\right)$, of the following type:

$$
u_{d}=\alpha_{d} U_{d, x_{d}}+v_{d}
$$

where as $d \rightarrow 0, \alpha_{d} \rightarrow Q_{\mathrm{m}}^{-1 /(p-1)},\left\|v_{d}\right\|_{d} \rightarrow 0$ and $x_{d} \rightarrow x_{\circ} \in \mathcal{M}$.

Proof. In view of Lemmas 10 and 11 we conclude that

$$
\#\left\{x \in \mathcal{M}_{\varrho}: D F(x)=0\right\} \geq \operatorname{cat}_{\mathcal{M}_{\varrho}}\left(F^{a_{d}}\right) \geq \operatorname{cat}_{\mathcal{M}_{\varrho}}(\mathcal{M})
$$

and the result readily follows. 
Appendix. We only describe the main steps of the proof of Proposition 1 and for more details we refer to the paper [11]. Let $\Phi_{P_{d}}$ be the mapping defined in Section 2 associated with $P_{d} \in \partial \Omega$, where $u_{d}$ attains its maximum on $\bar{\Omega}$. In what follows we assume $P_{d}=P$ and write $\psi, \Phi, \Phi_{j}, \Psi, \Psi_{j}$ instead of $\psi_{P_{d}}, \Phi_{P_{d}}, \Phi_{P_{d}, j}, \Psi_{P_{d}}, \Psi_{P_{d}, j}$, respectively. We assume that $\Phi$ is defined on an open set containing the closed ball $\bar{B}_{3 k}=\overline{B(0,3 k)}$, where $k>0$ is a small constant. We set

$$
w_{d}(z)= \begin{cases}u_{d}(\Phi(d z)) & \text { for } z \in \bar{B}_{3 k / d}^{+} \\ u_{d}\left(\Phi\left(d z^{\prime},-d z_{N}\right)\right) & \text { for } z \in \bar{B}_{3 k / d}^{-}\end{cases}
$$

As in [12] we check that $w_{d} \rightarrow w_{M}$ in $C_{\mathrm{loc}}^{2}\left(\mathbb{R}^{N}\right)$. We need a first order approximation in $d$ of $w_{d}$. Towards this end we set

$$
w_{d}(z)=w_{M}(z)+d \widetilde{w}_{d}(z) .
$$

The function $w_{d}$ satisfies the equation

$$
\sum_{i, j=1}^{N} a_{i j}^{*}(z) \frac{\partial w_{d}}{\partial z_{i} \partial z_{j}}+d \sum_{j=1}^{N} b_{j}^{*}(z) \frac{\partial w_{d}}{\partial z_{j}}-w_{d}+Q(\Phi(d z)) w_{d}^{p}=0
$$

in $\bar{B}_{3 k / d}$, where the coefficients $a_{i j}^{*}$ and $b_{j}^{*}$ are expressed in terms of the derivatives of $\Psi$ (see p. 835 in [12]), and moreover,

$$
\frac{\partial w_{d}}{\partial z_{N}}\left(z^{\prime}, 0\right)=0 \quad \text { for }\left|z^{\prime}\right|<\frac{3 k}{d} .
$$

Let $\chi \in C^{2}(\mathbb{R})$ be a cut-off function satisfying $0 \leq \chi(t) \leq 1$ on $\mathbb{R}, \chi(t)=0$ if $|t| \geq 3 / 2$ and $\chi(t)=1$ if $|t|<1$. We set $\chi_{R}(z)=\chi(|z| / R)$ for $z \in \mathbb{R}^{N}$ and $W_{R}(z)=\chi_{R}(z) w_{d}(z)$. We see that $W_{R} \in C^{2}\left(\mathbb{R}^{N}\right), W_{R}(z)=w_{d}(z)$ on $B_{R}$ and $W_{R}(z)=0$ on $\mathbb{R}^{N}-B_{2 R}$. From now on we assume that $R=k / d$. We let $d \phi_{R}(z)=W_{R}(z)-w_{M}(z)$, and we see that $\phi_{R}(z)=\widetilde{w}_{d}$ on $B_{k / d}$. The function $\phi_{R}$ satisfies the equation

$$
L \phi_{R}+A_{R} \phi_{R}+g_{R}+h\left(\phi_{R}\right)=0
$$

where

$$
\begin{aligned}
L & =\Delta-1-p M w_{M}^{p-1}, \\
g_{R} & =\frac{1}{d} A_{R} w_{M}-\frac{1}{d}\left(\Delta \chi_{R}+A_{R} \chi_{R}\right) w_{d}-\frac{1}{d} \sum_{i, j=1}^{N}\left(a_{i j}^{*}+a_{j i}^{*}\right) \frac{\partial \chi_{R}}{\partial z_{i}} \frac{\partial w_{d}}{\partial z_{j}}, \\
h\left(\phi_{R}\right) & =\frac{1}{d}\left[Q(\Phi(d z)) \chi_{R} w_{d}^{p}-M w_{M}^{p}+p M w_{M}^{p-1} d \phi_{R}\right],
\end{aligned}
$$


and

$$
\begin{aligned}
A_{R}= & d \chi_{2 R}(z)\left(2\left|z_{N}\right| \sum_{i, j=1}^{N-1} \psi_{d, i j} \frac{\partial^{2}}{\partial z_{i} \partial z_{j}}-\alpha_{d} \operatorname{sgn} z_{N} \frac{\partial}{\partial z_{N}}\right) \\
& +\chi_{3 R}\left(\sum_{i, j=1}^{N-1} \alpha_{i j}(z) \frac{\partial^{2}}{\partial z_{i} \partial z_{j}}+d \sum_{j=1}^{N} \beta_{j}(z) \frac{\partial}{\partial z_{j}}\right)
\end{aligned}
$$

where

$$
\psi_{d, i j}=\frac{\partial^{2} \psi_{d}}{\partial z_{i} \partial z_{j}}(0) \quad \text { and } \quad \alpha_{d}=\Delta \psi_{d}(0) .
$$

Using Lemmas 4.2 and 4.3 from [11] we can formulate the existence result for the following problem:

$$
\begin{gathered}
\Delta \phi-\phi+M w_{M}^{p-1} \phi+2\left|z_{N}\right| \sum_{i, j=1}^{N-1} \psi_{d, i j} \frac{\partial^{2} w_{M}}{\partial z_{i} \partial z_{j}} \\
\quad-\alpha_{d}\left(\operatorname{sgn} z_{N}\right) \frac{\partial w_{M}}{\partial z_{N}}=0 \quad \text { in } \mathbb{R}^{N}, \\
\phi(z) \rightarrow 0 \quad \text { as }|z| \rightarrow \infty .
\end{gathered}
$$

Proposition $\mathrm{A}_{1}$. Problem $\left(\mathrm{A}_{3}\right)-\left(\mathrm{A}_{4}\right)$ has a unique solution $\phi \in C^{2}\left(\mathbb{R}^{N}\right)$ satisfying

$$
\int_{\mathbb{R}^{N}} \phi \frac{\partial w_{M}}{\partial z_{j}} d z=0 \quad \text { for } j=1, \ldots, N .
$$

Furthermore, $\phi$ decays exponentially at infinity:

$$
|\phi(z)| \leq C e^{-\mu|z|} \quad \text { for } z \in \mathbb{R}^{N},
$$

for some constants $C>0$ and $\mu>0$ independent of $d$. The function $\phi$ is even in $z_{N}$ and

$$
\lim _{d \rightarrow 0} d^{-1} \sup _{z \in B_{k / d}}\left|w_{d}(z)-\left(w_{M}(z)+d \phi(z)\right)\right|=0 .
$$

Sketch of proof. Since $w_{d}(z)-\left(w_{M}(z)+d \phi(z)\right)=d\left(\phi_{R}(z)-\phi(z)\right)$ on $B_{R}$, for the proof of the last assertion $\left(\mathrm{A}_{5}\right)$ it is sufficient to show that $\sup \left|\phi_{R}(z)-\phi(z)\right| \rightarrow 0$ as $R \rightarrow \infty$. This is established by writing the following decomposition:

$$
\phi_{R}(z)=\sum_{j=1}^{N} a_{j}(R) \phi_{j}(z)+\zeta_{R}(z)
$$

where

$$
\phi_{j}(z)=c_{\circ} \frac{\partial w_{M}}{\partial z_{j}}, \quad c_{\circ}=\left(\frac{N}{\int_{\mathbb{R}^{N}} w_{M}^{\prime}(|z|)^{2} d z}\right)^{1 / 2}
$$


and showing that $a_{j}(R) \rightarrow 0$ and $\zeta_{R}(z) \rightarrow \phi$ as $R \rightarrow \infty$ (see Lemmas 4.7-4.9 in [11]).

Now we are in a position to establish the asymptotic formula (18) for $c_{d}$. Multiplying $\left(\mathrm{A}_{3}\right)$ by $w_{M}$ and integrating over $\mathbb{R}^{N}$ we obtain

$$
\begin{aligned}
\int_{\mathbb{R}_{+}^{N}}(p-1) M w_{M}^{p} \phi d z= & 2 \gamma \alpha_{d} M^{-2 /(p-1)} \\
& +\alpha_{d} M^{-2 /(p-1)} \int_{\mathbb{R}_{+}^{N}} w_{1} \frac{\partial w_{1}}{\partial z_{N}} d z
\end{aligned}
$$

(for details see Lemma 3.1 in [11]). Since $w_{d}$ satisfies (1) we have, using formula $\left(\mathrm{A}_{3}\right)$ from [12],

$$
\begin{aligned}
c_{d}= & \int_{\Omega} \frac{p-1}{2(p+1)} Q(x) u_{d}^{p+1} d x \\
= & \int_{D_{1}} \frac{p-1}{2(p+1)} Q(x) u_{d}^{p+1} d x+\int_{\Omega-D_{1}} \frac{p-1}{2(p+1)} Q(x) u_{d}^{p+1} d x \\
= & \int_{D_{1}} \frac{p-1}{2(p+1)} Q(x) u_{d}^{p+1} d x+O\left(e^{-\mu / d}\right) \\
= & d^{N} \int_{B_{k / d}^{+}} \frac{p-1}{2(p+1)} w_{d}(z)^{p+1} M\left(1-\alpha_{d} d z_{n}+O\left(d^{2}|z|^{2}\right)\right) \\
& +\int_{D_{1}} \frac{p-1}{2(p+1)}(Q(x)-M) u_{d}^{p+1} d x+O\left(e^{-\mu / d}\right) \\
= & I_{1}+I_{2}+O\left(e^{-\mu / d}\right) .
\end{aligned}
$$

We estimate $I_{1}$ using Proposition $\mathrm{A}_{1}$ :

$$
\begin{aligned}
I_{1}= & \frac{p-1}{2(p+1)} d^{N} \int_{B_{k / d}^{+}} M w_{d}(z)^{p+1}\left(1-d \alpha_{d} z_{N}\right) d z+d^{N+2} \\
= & \frac{p-1}{2(p+1)} d^{N} \int_{B_{k / d}^{+}} M\left(w_{M}+d(\phi+o(1))\right)^{p+1}\left(1-d \alpha_{d} z_{N}\right) d z+O\left(d^{N+2}\right) \\
= & d^{N}\left[\frac{p-1}{2(p+1)} \int_{B_{k / d}^{+}} M w_{M}^{p+1} d x+\frac{d}{2}(p-1) \int_{B_{k / d}^{+}} M w_{M}^{p} \phi d x\right. \\
& \left.-\alpha_{d} \frac{p-1}{2(p+1)} \int_{B_{k / d}^{+}} M w_{M}^{p+1} z_{N} d z+o(d)\right]
\end{aligned}
$$




$$
\begin{aligned}
= & d^{N}\left[\frac{p-1}{2(p+1)} \int_{\mathbb{R}_{+}^{N}} M w_{M}^{p+1} d z+\frac{d}{2}(p-1) \int_{\mathbb{R}_{+}^{N}} M w_{M}^{p} \phi d z\right. \\
& \left.-\frac{p-1}{2(p+1)} \alpha_{d} d \int_{\mathbb{R}_{+}^{N}} M w_{M}^{p+1} z_{N} d z+o(1)\right] .
\end{aligned}
$$

It then follows from $\left(\mathrm{A}_{6}\right)$ and an obvious modification of formula (3.13) in [12] that

$$
I_{1}=d^{N}\left\{\frac{1}{2} I_{M}\left(w_{M}\right)-\gamma d \alpha_{d} M^{-2 /(p-1)}\right\}+o(1) d^{N} .
$$

Finally, using (17) we check that $I_{2}=o(1) d^{N}$ and the result follows.

Acknowledgements. P.J.W. and J.F.Y. were supported by ARC Small Grants Scheme.

\section{References}

[1] A. Ambrosetti and P. Rabinowitz, Dual variational methods in critical point theory and applications, J. Funct. Anal. 14 (1973), 349-381.

[2] A. Bahri and M. Coron, On a nonlinear elliptic equation involving the Sobolev exponent: the effect of the topology of the domain, Comm. Pure Appl. Math. 41 (1988), 253-294.

[3] J. Chabrowski and S. S. Yan, The effect of the graph topology on a semilinear elliptic equations with critical exponents, Topol. Methods Nonlinear Anal. 12 (1998), 1-26.

[4] N. Dancer and S. S. Yan, Multipeak solutions for a singularly perturbed Neumann problem, Pacific J. Math. 189 (1999), 241-262.

[5] A. Gierer and H. Meinhardt, A theory of biological pattern formation, Kybernetik 12 (1972), 30-39.

[6] E. Keller and A. Segel, Initiation of slime model aggregation viewed as an instability, J. Theoret. Biol. 26 (1976), 399-415.

[7] M. K. Kwong and L. Zhang, Uniqueness of positive solutions of $\Delta u+f(u)=0$ in $\mathbb{R}^{n}$, Differential Integral Equations 4 (1991), 583-599.

[8] Y. Y. Li, On a singularly perturbed equation with Neumann boundary conditions, Comm. Partial Differential Equations 3-4 (1998), 487-545.

[9] C. S. Lin, W. M. Ni and I. Takagi, Large amplitude stationary solutions to a chemotaxis system, J. Differential Equations 72 (1988), 1-27.

[10] W. M. Ni and I. Takagi, On the Neumann problem for some semilinear elliptic equations and systems of activator-inhibitor type, Trans. Amer. Math. Soc. 297 (1986), 351-368.

[11] - - - Locating the peaks of least energy solutions to a semilinear Neumann problem, Duke Math. J. 70 (1993), 247-281.

[12] - - - On the shape of least-energy solutions to a semilinear Neumann problem, Comm. Pure Appl. Math. 44 (1991), 819-851.

[13] O. Rey, The role of the Green's function in a non-linear elliptic equation involving the critical Sobolev exponent, J. Funct. Anal. 89 (1990), 1-52.

[14] Z. Q. Wang, On the existence of multiple, single-peaked solutions for a semilinear Neumann problem, Arch. Rational Mech. Anal. 120 (1992), 375-399. 
[15] J. Wei, On the boundary spike solutions to a singularly perturbed Neumann problem, J. Differential Equations 134 (1997), 104-133.

Department of Mathematics The University of Queensland Brisbane 4072, Qld, Australia E-mail: jhc@maths.uq.edu.au
Permanent address of J. F. Yang: Department of Mathematics Wuhan Institute of Physics and Mathematics Chinese Academy of Sciences P.O. Box 71010 Wuhan 430071, P.R. China

Current address of J. F. Yang: ECC-UNICAMP 13083-970, Campinas, S.P. Brazil 